University of Nebraska - Lincoln

DigitalCommons@University of Nebraska - Lincoln

$7-2020$

\title{
Understanding the Impact of Room Acoustic Conditions on the Kurtosis Levels of Various Noise Signals
}

William Spallino

University of Nebraska - Lincoln, wspallino@huskers.unl.edu

Follow this and additional works at: https://digitalcommons.unl.edu/archengdiss

Part of the Architectural Engineering Commons

Spallino, William, "Understanding the Impact of Room Acoustic Conditions on the Kurtosis Levels of Various Noise Signals" (2020). Architectural Engineering -- Dissertations and Student Research. 60. https://digitalcommons.unl.edu/archengdiss/60

This Article is brought to you for free and open access by the Architectural Engineering and Construction, Durham School of at DigitalCommons@University of Nebraska - Lincoln. It has been accepted for inclusion in Architectural Engineering -- Dissertations and Student Research by an authorized administrator of DigitalCommons@University of Nebraska - Lincoln. 


\title{
UNDERSTANDING THE IMPACT OF ROOM ACOUSTIC CONDITIONS ON KURTOSIS LEVELS OF VARIOUS NOISE SIGNALS
}

by

William Jesse Spallino

\author{
A THESIS \\ Presented to the Faculty of \\ The Graduate College at the University of Nebraska \\ In Partial Fulfillment of Requirements \\ For the Degree of Master of Science
}

Major: Architectural Engineering

Under the Supervision of Professor Lily M. Wang

Lincoln, Nebraska

July 2020 (Updated December 2020) 


\title{
UNDERSTANDING THE IMPACT OF ROOM ACOUSTIC CONDITIONS ON THE KURTOSIS LEVELS OF VARIOUS NOISE SIGNALS
}

\author{
William Jesse Spallino, M.S. \\ University of Nebraska, 2020
}

Adviser: Lily M. Wang

Impulsive noise can be common in certain occupational and recreational settings, such as manufacturing plants, construction sites, and firing ranges. While many regulations and guidelines for noise exposure exist, their mathematical basis is stronger for continuous noise, and concerns have been raised about the possibility that impulsive noise may be more harmful to people's hearing than those methods would let on. Much work has been done on establishing metrics that accurately assess the severity and hearing risk associated with impulsive noise, but the effects of room acoustic conditions on those metrics have been heretofore understudied.

This study calculated room impulse response-based metrics of rooms' acoustical properties and the kurtosis levels (a metric which has been proposed and vetted during the last two decades) for four different noise signals. The impact of rooms' sound absorption and sound scattering properties on those kurtosis levels is presented and supported with statistical analysis. Due to a low number of data points, no results were obtained that could support a firm statement on the phenomena under examination, but several categories approached statistical significance; further investigation is recommended. 


\section{COPYRIGHT}

Copyright 2020, William Jesse Spallino 


\section{Acknowledgements}

\section{University of Nebraska - Lincoln}

- Dr. Lily Wang

- Jared Paine

I thank Dr. Wang for her assistance as my academic adviser. Without her support, the project's analytical complexities would have taken much greater time and effort to overcome. Additionally, her support for my chosen topic despite it falling outside our research group's usual expertise was invaluable, and her choice of assistantship for my financial aid package forged a connection with another research group that proved very valuable to the project. Thanks to her support, I was able to present the project detailed in this thesis to the Acoustical Society of America conference "Acoustics Virtually Everywhere," in December 2020. I thank Mr. Paine for assisting with the refinement of room impulse response measurements, to the extent of carrying out measurements on my behalf in some cases.

\section{National Institute for Occupational Safety and Health}

- Capt. William Murphy, PhD

- Capt. Chucri Kardous, MSEE, PE

- Cdr. Edward Zechmann, PhD

Cdr. Zechmann's work is the direct motivation for this paper, and I thank him for his kindness in granting me direct access to his dissertation so that I could start my literature review from a more informed position. I thank Capt. Murphy and Capt. Kardous for their assistance in developing the scope of this project, identifying the gap in the body of knowledge that it serves to begin filling, and for putting me in touch with Cdr. Zechmann.

\section{Boys Town National Research Hospital}


- Dr. Daniel Rasetshwane

- Dr. Chris Stecker

I was placed in contact with the hearing researchers at Boys Town National Research Hospital (BTNRH hereafter) through a graduate assistantship funded by the National Institute of General Medical Sciences under grant number P20GM109023, and it was Dr. Rasetshwane who first showed me the importance of impulsive noise by discussing how prominently it figured into the hearing problems of many participants in his research. After I had defined the topic of this thesis, he provided assistance in scheduling measurements in an audiovisual lab under his partial supervision. Dr. Stecker allowed me to take reference data for the project in the anechoic chamber he supervises at BTNRH, and I am very grateful for that assistance as well. 


\section{Table of Contents}

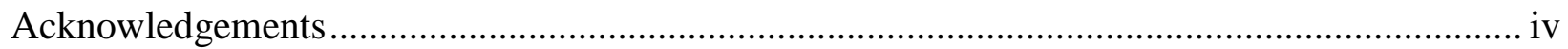

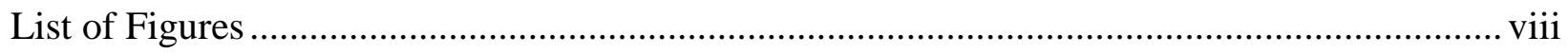

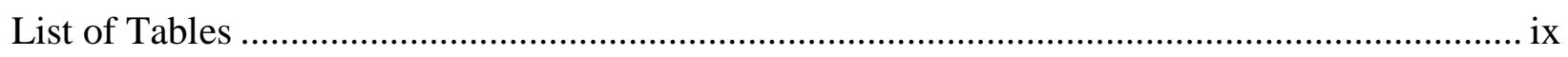

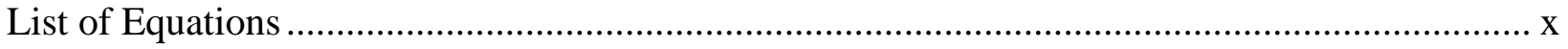

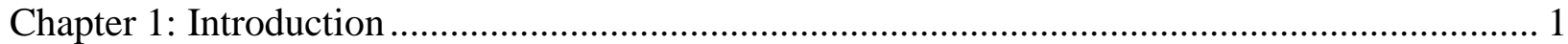

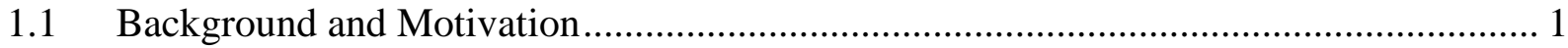

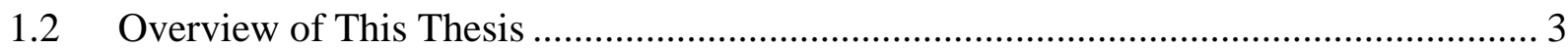

Chapter 2: Review of Previous Literature ............................................................................ 5

Chapter 3: Methods and Experimental Considerations ..................................................... 12

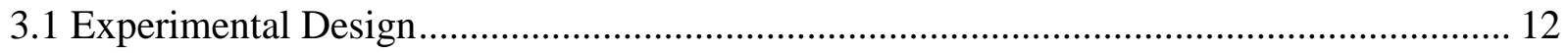

3.1.1 Data Collection Considerations ...................................................................... 12

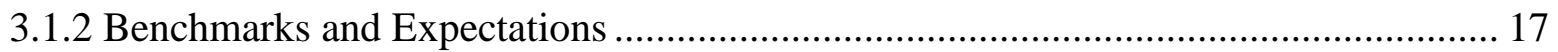

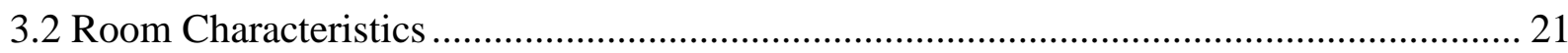

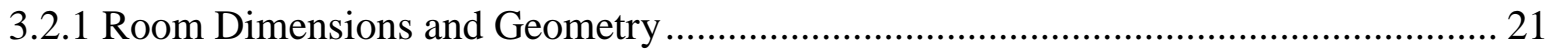

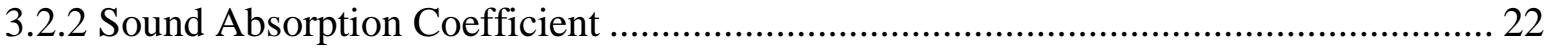

3.2.3 Number of Peaks in the Impulse Response ......................................................... 25

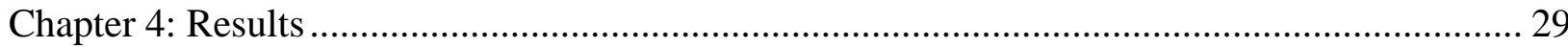

4.1 Room Properties and Signals of Interest..................................................................... 29

4.1.1 Room Absorption Coefficients ........................................................................ 29

4.1.2 Numbers of Peaks in Room Impulse Responses................................................ 32

4.1.3 Signals of Interest and Predictions about their Behavior......................................... 33

4.2 Kurtosis Levels with respect to Absorption Coefficient ............................................... 35

4.3 Kurtosis Levels with respect to Number of Peaks ...................................................... 37

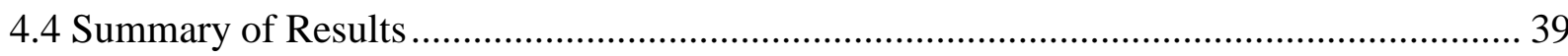

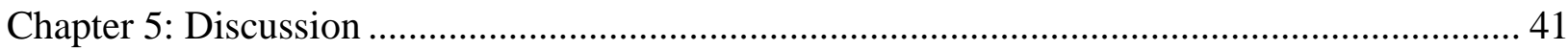

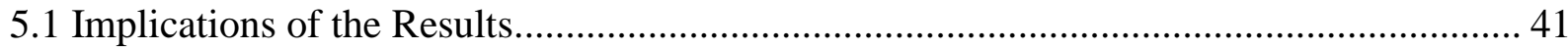

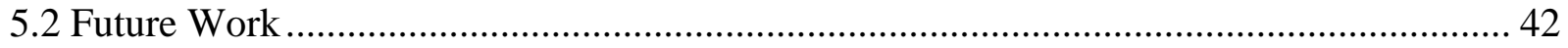

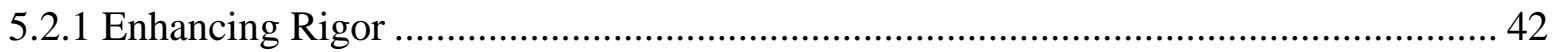

5.2.2 Connections with Other Disciplines ............................................................. 44

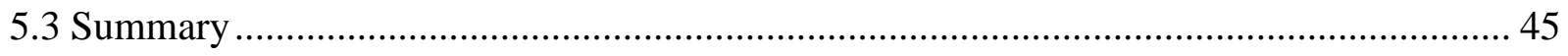

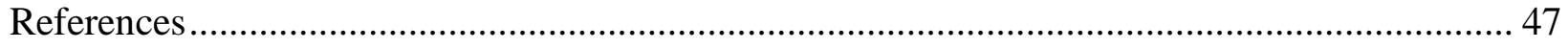


Appendix A: MATLAB Code

A-1: Absorption Coefficient Calculation from Room Geometry and Reverberation Time (getAbsorptionCoef.m)

A-2: Normalized Number of Peaks Calculation from Room Impulse Response

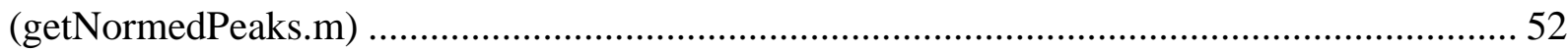

A-3: Kurtosis Level Calculation from Convolution (getConvolvedLkFourSignals.m) .......... 54

Appendix B: Discussion of Original Methodology ................................................................ 56 


\section{List of Figures}

Figure 3.1: Photograph of a source-receiver pair in the Strauss Performing Arts Center recital hall.

Figure 3.2: Photograph of the omnidirectional loudspeaker used for room impulse response measurements in a common talker position in Peter Kiewit Institute room 150 17

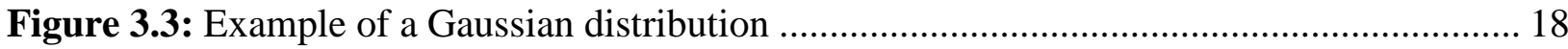

Figure 3.4: Example of an arcsine distribution....................................................................... 19

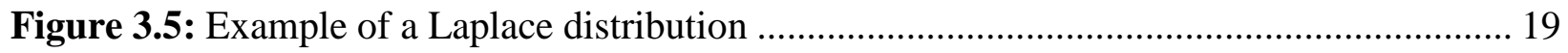

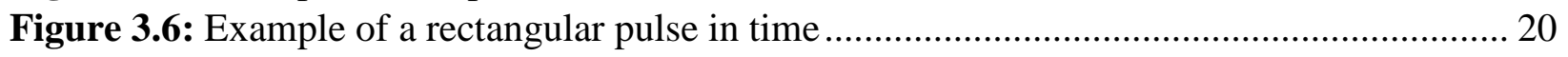

Figure 3.6: Example of strongly absorptive surfaces .................................................... 23

Figure 3.7: Example of a surface designed to scatter sound................................................. 26

Figure 3.8: The early portion of the Strauss Performing Arts Center Recital Hall's impulse response, transformed using Bliefnick's methodology ................................................... 28

Figure 4.1: Graphic showing the changes in kurtosis level of Laplacian noise as the duration of the computer-generated signal increases .................................................................. 34

Figure 4.2: Kurtosis levels of all signals of interest plotted against absorption coefficient ........ 36

Figure 4.3: Kurtosis levels of all signals of interest plotted against number of peaks .............. 38 


\section{List of Tables}

Table 4.1: Reverberation-related information for each room ........................................... 30

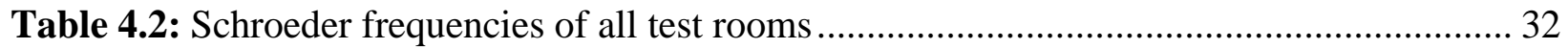

Table 4.3: Normalized number of peaks in each room's impulse response ............................ 33

Table 4.4: Statistical summary of kurtosis levels with respect to absorption coefficient........... 39

Table 4.5: Statistical summary of kurtosis levels with respect to number of peaks .................. 39 


\section{List of Equations}

Equation 1: Formula for calculating the kurtosis level ............................................................ 9

Equation 2: Formula for transforming uniformly distributed random numbers to follow the Laplace distribution ....................................................................................................... 15

Equation 3: Formula for estimating room critical distance ……………………………........... 21

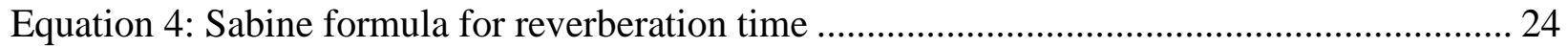

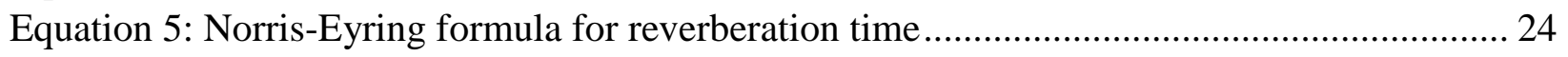

Equation 6: Formula for room Schroeder frequency …………………………………............ 31 


\section{Chapter 1: Introduction}

\subsection{Background and Motivation}

Noise-induced hearing loss is a well-known problem in both the public consciousness and in legal regulations. Means of preventing and mitigating it are codified by entities like the United States' Occupational Safety and Health Administration (OSHA) and National Institute for Occupational Safety and Health (NIOSH), whose contributions are discussed more thoroughly in Chapter 2. However, research is continually undertaken to improve various aspects of how society addresses noise-induced hearing loss, as evolving understanding can lead to better preventative measures. A particular kind of noise, impulsive noise, has been getting significant attention in recent years, and gave rise to this study.

Impulsive noise is comprised of two categories: impulse noise (associated with explosive discharge) and impact noise (associated with colliding objects). Both categories share the defining trait of having a rapid onset from ambient sound pressure to a high peak level before decaying; the theoretical limit of this behavior would be a Dirac delta function. Practical examples of this kind of noise include a single strike of a snare drum, a handclap, a single gunshot, and an explosion (which would be mathematically modeled by a Friedlander waveform). Impulsive noise is especially prevalent in certain occupational settings, such as the military and some manufacturing plants, which makes understanding it especially important for ensuring the health and safety of personnel working in those areas. Based on decades of studies involving both human and animal auditory systems, impulsive noise's effects on the human auditory system may be even worse than exposure to continuous noise (Thiery and Meyer-Bisch 1988). Its inherently high pressure amplitudes can create acute effects in addition to the usual 
harmful effects of long-term or chronic noise exposure, possibly complicating them. All these reasons and more have led to an increase in the research and attention being dedicated to impulsive noise in recent years.

In the existing literature, impulsive noise has been repeatedly identified as an area where health risk assessment ought to be improved, as it does not behave well under commonly used noise control frameworks and is therefore difficult to model accurately. Attempts have been made at creating models for impulsive noise's hearing loss risk, with the most notable including the Auditory Hazard Assessment Algorithm for Humans (hereafter abbreviated "AHAAH model") laid out in MIL-STD-1474E (U.S. Department of Defense 2015) and models based on the audiological equal-energy hypothesis, such as that laid out by Atherley and Martin (1967). Challenges and issues have arisen for each attempt, however, upon deeper investigation. Deficient modeling of impulsive noise leaves too much unknown when assessing its associated risk of hearing loss, so research has been undertaken in an effort to improve the quantification and modeling of impulsive noise (Flamme and Murphy 2020). Related research has discovered promise in the concept of treating noise exposures as distributions of sound pressure and taking cues from their descriptive statistics (Zhao et al. 2010; Davis et al. 2012; Xie et al. 2016). In particular, the conversion of a sound pressure distribution's kurtosis into a metric in decibels has appeared to correlate well with the measured loss of hearing in several data sets spanning human workers and animal trial groups. This study seeks to understand the effect of changes in room sound absorption and room sound scattering properties on that "kurtosis level" metric. 


\subsection{Overview of This Thesis}

Chapter 1 of this thesis summarizes the context and motivation surrounding this study. Subsequent chapters address the methods used to perform the research this thesis presents, the data collected and analyzed during that research, and the potential implications of those results. Also included is an appendix which provides the MATLAB code written by the author for purposes of data analysis, as well as a shorter second appendix which discusses a methodology that was attempted in the early stages of the study.

Chapter 2 discusses the previous literature that informed and, in some cases, motivated this study in greater depth. They are presented in order of how directly they motivated this study, with chronological order acknowledged within that structure.

Chapter 3 details the methodologies of this study. Due to the limitations on time and scope inherent to a masters-level project, it was decided that a direct test of the relevant predictions made by previous researchers would be carried out. To that end, various rooms were tested that had different absorption and scattering characteristics (as calculated from their impulse responses). Computer-generated noise signals with known expected kurtosis levels were then convolved with the rooms' impulse responses to produce room-specific versions of the signals that could be used to "measure" kurtosis levels. Correlation coefficients and p-values were also calculated for purposes of determining the trends, if any, that appeared in the data with respect to sound absorption coefficient and the study's chosen scattering metric, number of peaks in the impulse response, and the significance level of such trends. 
Chapter 4 details the numerical results of the study. In the course of doing so, the chapter also reiterates certain experimental considerations from Chapter 3 to give context to the data presented.

Chapter 5 discusses the study in retrospect, its outcomes, and the conclusions that could be drawn from its results. It also presents some potential concepts and considerations for future studies that might deepen and solidify our understanding of room acoustics' interplay with the severity of noise-induced trauma, and of impulsive noise in general.

MATLAB was the primary analysis tool for this study, and was used not only to carry out statistical operations and data processing, but also to create the graphs presented in this thesis. Appendix A contains all the self-written code used during data analysis. Comments written with the code are left intact so as to minimize the amount of text besides the programs themselves, but each entry is accompanied by a reference to tables and graphs in this thesis that owe some part of their contents to that program.

Appendix B presents a brief discussion of a more equipment- and effort-intensive methodology that was considered and used in the early stages of the study. This methodology was abandoned, both because it produced low-quality data and made the study less efficient. 


\section{Chapter 2: Review of Previous Literature}

Research into how to better assess the hearing risks posed by exposure to impulsive noise has made many strides. Before that discussion may begin, though, it is important to consider the current measures that are in place to protect people's hearing as they go about their lives.

Perhaps the first sub-category to spring to mind when discussing noise-induced hearing loss is occupational noise exposure, with both the U.S. Occupational Safety and Health Administration (OSHA) and the U.S. National Institute for Occupational Safety and Health (NIOSH) issuing guidelines within their respective scopes (U.S. Department of Labor 2008; U.S. National Institute for Occupational Safety and Health 1998). OSHA in particular states that any exposure of 115 A-weighted decibels (abbreviated dBA, referring to a system of weighting sound pressure levels in relation to their octave bands so they better reflect the response of the human auditory system) is only allowable if its total duration for a workday is fifteen minutes or less, making no allowance for exposure to higher noise levels.

Other research has determined that high levels of non-occupational noise, such as the sounds generated by car traffic, can have harmful effects on people's physical and mental health (de Paiva Vianna, Rodrigues, and Alves Cardoso 2015). While these studies are not necessarily conducted under the aegis of any regulatory entity, the problems they observe nevertheless are addressed in legislation, as municipalities have been known to place restrictions on the noise which can be generated during certain hours and/or by certain sources. For example, the city of Omaha, Nebraska, where this study was conducted, restricts vehicle noise based on the speed limit of the street in question and the type of vehicle emitting the noise (overall, it never allows vehicle noise to exceed $90 \mathrm{dBA}$ at 50 feet away from the source); loud construction equipment 
such as pile drivers that produce "loud or unusual noise" are not permitted to operate between the hours of 10:00 pm and 7:00 am local time under a different section of the code (Officials of the City of Omaha, Nebraska 1996).

Notably, in many cases, the restrictions on noise are put in terms of equivalent sound pressure levels. Those levels describe a continuous, non-varying noise exposure that would impart equal sound energy to that of the measured noise exposure (Long 2014). Depending on the settings of the sound level meter or dosimeter which is collecting data, the high spikes of short-term energy uniquely generated by impulsive noise (or impulsive components of a complex noise signal) may not appear to their full extent in the equivalent sound pressure level metric. This restricts those metrics' ability to describe the risk associated with impulsive noise, especially when it is part of a more complex sonic environment (as it would be at a roadside construction site, for example). Therefore, a dedicated metric for impulsive noise can help in tailoring hearing loss prevention and hearing conservation programs to situations in which it is prevalent. This observation has been made in many studies of impulsive noise, some of which date back decades (Sulkowski, Kowalska, and Lipowczan 1983; Patterson 1991; Goley, Song, and $\operatorname{Kim} 2011)$.

The literature that explores, explains, and specifies the need for methods, metrics, and systems that deal specifically with impulsive noise has been building for a long time; however, it also has great breadth, spanning areas like audiology, noise control, psychoacoustics, and signal processing. Some of those areas deserve special mention, as they either provide perspective on the topics this study seeks to address or directly support arguments which form the foundation of this study. For example, one of the first and most persistent problems observed by the body of work is that the peak sound pressure levels of impulsive noise can frequently exceed the limits of 
applicability stated in standards, damage risk criteria (abbreviated DRC), and most glaringly the physical limitations of sound measurement equipment prescribed in such documents. This issue has been reaffirmed several times, as in Kardous and Willson (2004), whose research informed recommended improvements to dosimeters such that they could recognize the severity of an impulsive noise environment (Kardous, Willson, and Murphy 2005); meanwhile, it was seemingly ignored by a set of DRC proposed contemporaneously with the early stages of research into impulsive noise (Ward 1968). The Berger et al "Noise Navigator" database shows this egregious difference in peak levels for such sources as an activated motorboat engine and revolver shots (2015); a study by Kamerer et al (2019) uses those particular data points and a weighting system designed by the study's authors to make the extended argument that impulsive noise exposure can be much more deleterious to individuals' auditory health than many conventional sound measurement metrics might indicate. These facts create an urgent need for an improved noise analysis model.

As part of the drive to create such a model, several studies have been dedicated to the difference in impact on people's auditory health between impulsive noise and continuous noise. These studies have ranged from comparing occupational noise exposures in different industries (Sulkowski, Kowalska, and Lipowczan 1983) to exploring the hearing threshold shifts and eventual hearing loss due to specific kinds of impulsive or partly impulsive noise exposure (Hamernik et al. 1994; Thiery and Meyer-Bisch 1988). Although some studies acknowledge that temporary threshold shifts are recovered in the short term, many find that the risk of long-term hearing damage due to impulsive noise exposure is higher than the risk of such damage due to exposure to continuous/non-impulsive noise (Coles et al. 1967; Zhao et al. 2010). The large 
quantity of such assertions lends extra urgency to the need to quantify the additional risk so it can be communicated and mitigated efficiently and appropriately.

Another significant topic in the field of impulsive noise research is the development of the mathematics that form an irreplaceable foundation for this thesis. Many studies by many researchers contributed to the determination that the kurtosis of the sound pressure distribution of a noise signal could indicate its degree of impulsiveness, some of which are discussed in detail here. Erdreich (1986), for one, published an important discussion on the merits of considering the measured sound pressures that make up a signal over time as a distribution and examining their descriptive statistics instead of other metrics that were under consideration at the time, such as the crest factor (the ratio of a signal's peak sound pressure to its root-mean-square sound pressure). Still other studies (Broch 1980; Dunn et al. 1991; Hamernik, Patterson, and Salvi 1986; Qiu, Hamernik, and Davis 2013) investigated the relationship between the sound pressure distributions of impulsive noises and the hearing damage sustained by those exposed to them, with the data under consideration spanning both human threshold shifts due to noise exposure and studies of the chinchilla's auditory system under exposure to impulsive noise.

In the area of defining metrics that demonstrate the contribution of a noise's impulsive character to the overall hearing risk it poses, previous work has considered many options. Early on, one popular view on the topic was based on an audiological equal-energy hypothesis; its premise was that noise exposures consisting of equal sound energy will ultimately inflict the same degree of harm to a person's auditory system (note the similarity in concept to an equivalent sound pressure level). Although support for this approach as applied to noise assessment has waned (Broch 1980; Patterson 1991; Roberto et al. 1985), some data suggest that it may inform a more appropriate audiological exchange rate for situations involving impulsive 
noise (Suter 2017). The AHAAH model (Price 2007a; 2007b) attempts to quantify the risk associated with exposure to impulsive noise, but has been challenged by research into the accuracy of its predictions (Flamme et al. 2016; McGregor et al. 2017; Zagadou et al. 2016; Deiters et al. 2019; Jones, Greene, and Ahroon 2019). A foundational argument of this study supported by recent research, by contrast, is that the A-weighted equivalent sound pressure level $(\mathrm{dBA})$ is a viable starting point for capturing the risks associated with impulsive noise numerically. According to a report published by NIOSH (Murphy and Kardous 2012), that metric is viable for several reasons, including its preexisting status as a "go-to" metric in the field of acoustics and the fact that it correlates as well as or better than any other metric under consideration with damage to the auditory system due to impulsive noise.

After the determination was made that the A-weighted equivalent sound pressure level was a suitable base metric, research on this topic focused on investigating various corrections, companions, and other modifications for it that would allow the mathematics to capture the real risk of impulsive noise. Those investigations combined with the knowledge generated by researchers who advocated for the treatment of noise exposures as distributions of sound pressure led to the creation and continuing validation of the kurtosis level metric (Lei, Ahroon, and Hamernik 1994; Goley, Song, and Kim 2011). Its form is supported by empirical data collected by Qiu et al (2006) that implied that the sample kurtosis of sound pressure distributions was the quantity that best predicted the response of and damage to chinchillas' auditory systems among the metrics they tested. It considers the sound of interest as a distribution of sound pressures and calculates its kurtosis, a measure of how likely it is that a random number from the distribution will be an outlier. The kurtosis is then converted into a quantity in decibels by means of Equation 1 below: 


$$
L_{k}=10 \log \left(\frac{k}{3}\right)
$$

where $k$ is the kurtosis of the set of sound pressures under consideration (Zechmann 2019). The division by three within the logarithm stems from the fact that any univariate normal distribution has a kurtosis of three, so a ratio greater than one implies high kurtosis, which in turn implies some degree of impulsive character.

The study that most directly inspired this research was conducted by Zechmann (2019), who developed understanding of how the kurtosis level metric characterizes sounds using signal processing methods. In the concluding sections of Zechmann's paper, it is noted that the variation in the kurtosis levels of sounds due to differences in room acoustics is yet to be fully understood; this study attempts to illuminate the probable answers to that question. Despite the gaps in understanding, some facts are known about the interplay between room acoustical factors and impulsive noise; for example, Murphy and Xiang (2019) created a model of an indoor firing range in the acoustic modeling software CATT and found that its $95 \%$ confidence interval was reasonable when compared to measured reverberation times in the real firing range, especially in the middle octave bands. However, the model noticeably overestimated the speech transmission index of the room and the Sabine and Norris-Eyring equations failed to match the room's measured reverberation times, suggesting that some combination of the firing range's geometry and the behavior of impulsive noise within its confines may confound traditional acoustical assessment methods. Thus, studying the effects of rooms' acoustical properties on the kurtosis level metric may complement the insights granted by modeling spaces that experience impulsive noise while in use. 
Zechmann also provides predictions regarding the kurtosis levels of certain sounds that are easy to characterize, statistically speaking. Those sounds are white noise (whose sound pressure distribution is expected to have Gaussian qualities), a pure tone (whose sound pressure distribution is expected to resemble an arcsine distribution), and impulsive noise (which is expected to behave as a generalized high-kurtosis distribution) (2019); example plots of each distribution can be found in Section 3.1.2. Those predictions proved immensely useful as a starting point for this study, and their details are discussed in greater detail in the following chapters. 


\section{Chapter 3: Methods and Experimental Considerations}

\subsection{Experimental Design}

This study was intended to examine the behavior of the kurtosis level metric, which was explained in Chapter 2, under changes to room acoustical properties. To be specific, those properties were sound absorption and sound scattering. To that end, both a data collection setup and a test environment had to be decided upon; this section details the decisions made and the rationale behind them.

\subsubsection{Data Collection Considerations}

First, the test environment came under consideration. The two primary options for setting up a testing environment were as follows: taking a single room as a test environment and varying its absorption and scattering properties through the addition and removal of acoustical products, or testing in multiple rooms and using their "natural" absorption and scattering properties as irregularly spaced data points. Ultimately, the latter was chosen due to restrictions on the time and money available to the study. In hopes of covering as wide a range as possible in both absorption and scattering (see later sections of this chapter for information on the specific metrics used), a set of rooms was selected for testing that had a variety of building materials, shapes, volumes, and usages. As seen in Chapter 4, this expectation was generally met. The specific rooms chosen, and their respective purposes, volumes, absorption coefficients, and numbers of peaks in the impulse response (see Section 3.2.3), are presented in Section 4.1. An additional reason for the importance of selecting rooms with a wide range of acoustical 
properties was that the greater the variety that was achieved, the more generalizable the results would be. Despite this being a consideration from the outset, success was only partial; see Section 5.2.1 for further discussion of this topic.

Second, the data collection apparatus and procedure were considered. While investigating how to obtain absorption and scattering metrics, the author discovered methods that made both calculable from the room's impulse response, so a setup for collecting that information was prepared. For this study's purposes, simply logging sound pressure levels over time with a sound level meter produced sufficient information about the background noise level in each room. Before any acoustical data could be collected, it was important to measure the rooms' dimensions so that the volume and surface area of each room could be calculated; this was done using a laser distance measurement device and the outcomes were recorded along with all other calculations done in the field. These calculations were combined with a rough estimate of the room reverberation time (obtained by listening to the room's response to a handclap and checking a test sine sweep's results) to estimate the acoustical critical distance, as well as applied to the calculation of other important metrics later in the analysis process. The critical distance estimate in particular, however, informed the placement of the source and receiver in each room. Once positioning was established, the sound level meter's microphone was pointed away from any nearby objects to avoid excess influence on its readings by reflections from their surfaces. Figure 3.1 below shows a source-receiver pair configured optimally.

The specific equipment used in measuring the room's impulse response included a laptop running EASERA room acoustics measurement software, an RME Babyface audio mixer, a Larson Davis power amplifier, a Larson Davis Model 831 sound level meter (Type 1) using a PCB $1 / 2$ " microphone, and an omnidirectional loudspeaker. A set of eight sine sweeps (plus one 
presend) was played through the omnidirectional loudspeaker to excite the room; the excitation and its associated reverberations were relayed through the sound level meter to the laptop, where EASERA automatically calculated the room's reverberation time and created a graph of the room impulse response, among other details like clarity index. The capture period for room impulse response data was intentionally set to be longer than the estimated reverberation time discussed earlier in this section, so that any excess "tail" could be trimmed rather than retaking entire measurements due to loss of meaningful information. The capture period itself was never longer than three seconds, although 1.5 seconds was the setting of choice in more absorptive spaces.

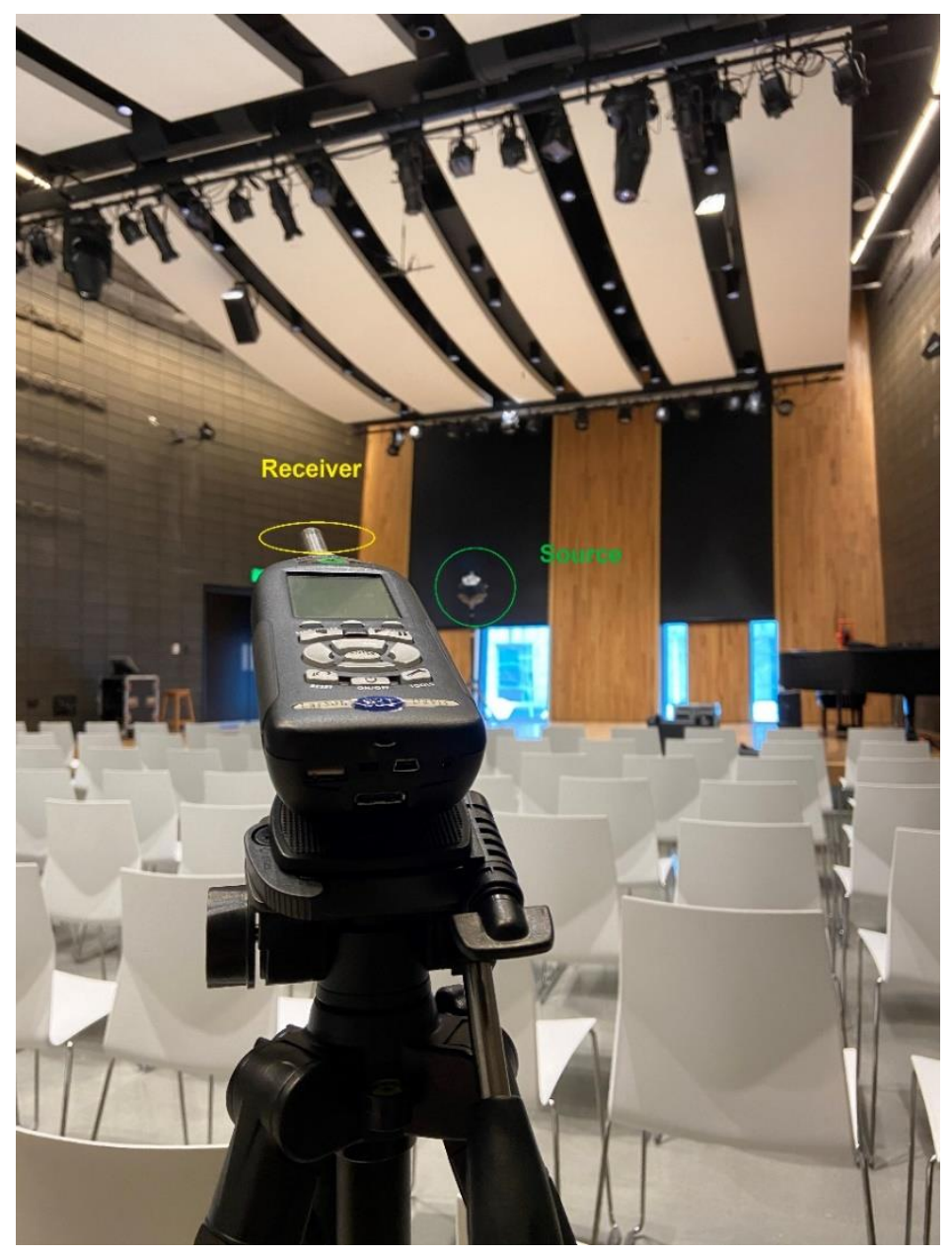

Figure 3.1: Photograph of a Larson-Davis Model 831 sound level meter being used in the Strauss Performing Arts Center recital hall (with omnidirectional loudspeaker just left of center in the background) 
The sound level meter was also used to record background noise levels during this portion of the procedure. In the final version of this protocol before the COVID-19 pandemic interrupted the optimization and polishing portion of data collection, the meter was set to use a fast response and capture a two-minute-long time history of sound pressure levels (unweighted, A-weighted, and in both octave bands and 1/3 octave bands) consisting of data points taken every 20 milliseconds; spectral analysis was disabled other than the collection of octave band and third-octave-band data and no triggers or day-night metrics were utilized. In order to achieve reasonable signal-to-noise ratios in certain rooms, the output gain of the sound level meter microphone had to be adjusted upwards.

Although an attempt was made to project the signals of interest from Zechmann's work into the rooms physically and measure them with the sound level meter, this procedure was abandoned (see Appendix B for full discussion). It left several important variables uncontrolled and could not achieve the same degree of precision as previous researchers did. For those reasons, it was decided to use the impulse response data for this portion of the analysis as well. Specifically, a self-written MATLAB program was used to convolve the room impulse response sound pressures with computer-generated versions of the signals of interest: white noise, a $1 \mathrm{kHz}$ sine wave serving as a pure tone, a series of rectangular impulses, and (by the author's choice in designing the study) a continuous signal whose sound pressures follow the Laplace distribution. The latter signal was transformed from uniformly distributed random numbers by means of Equation 2 below:

$$
L=-\operatorname{sgn}(U) * \ln (1-2|U|) .
$$

In Equation 2, $U$ is a random number chosen from the uniform distribution between -0.5 and 0.5 and $L$ is the corresponding random number belonging to the Laplace distribution with location 
parameter 0 and scale parameter 1 . The series of impulses and the Laplacian noise were both tied theoretically to impulsive noise, as the former is truly impulsive and the latter was this study's best means of simulating complex noise environments consisting of continuous noise with impulsive components (which are an important potential use case for the kurtosis level metric). This decision's implications are briefly discussed in Section 5.1, but it is worth mentioning now that this procedure does not fully address that potential use case, as it still assumes silence in the room under examination aside from the signal of interest.

Once the room impulse response and the signals of interest were convolved together, the kurtosis of each resulting waveform was calculated. That value, in turn, was fed into Equation 1 (see Chapter 2) to obtain the kurtosis level for that signal in that room. In all these calculations, the signals created in MATLAB matched EASERA's sampling rate of $44.1 \mathrm{kHz}$ and were five minutes (300 seconds) long. This length was chosen after a brief investigation of how the length of the generated signal impacted the resultant kurtosis level when convolved with an identical room impulse response; as Chapter 4 will show graphically, the kurtosis levels of all four signals of interest asymptotically approached a theoretical value consistent with the expectations laid out by Zechmann (see Section 3.1.2). Five minutes was the longest signal tested; any longer would have lessened the study's computational efficiency.

During data collection, the omnidirectional loudspeaker was located where a talker or other sound source for each room might be located during normal use in the author's best judgment. Examples include center stage in the Strauss Performing Arts Recital Hall and aligned with other sound sources in the Boys Town National Research Hospital AV Lab. The goal in doing so was to excite the room as similarly as possible to its excitation during normal 
use, as those conditions are what would create the sonic environment ultimately experienced by its occupants. For an example of this placement protocol in action, see Figure 3.2.

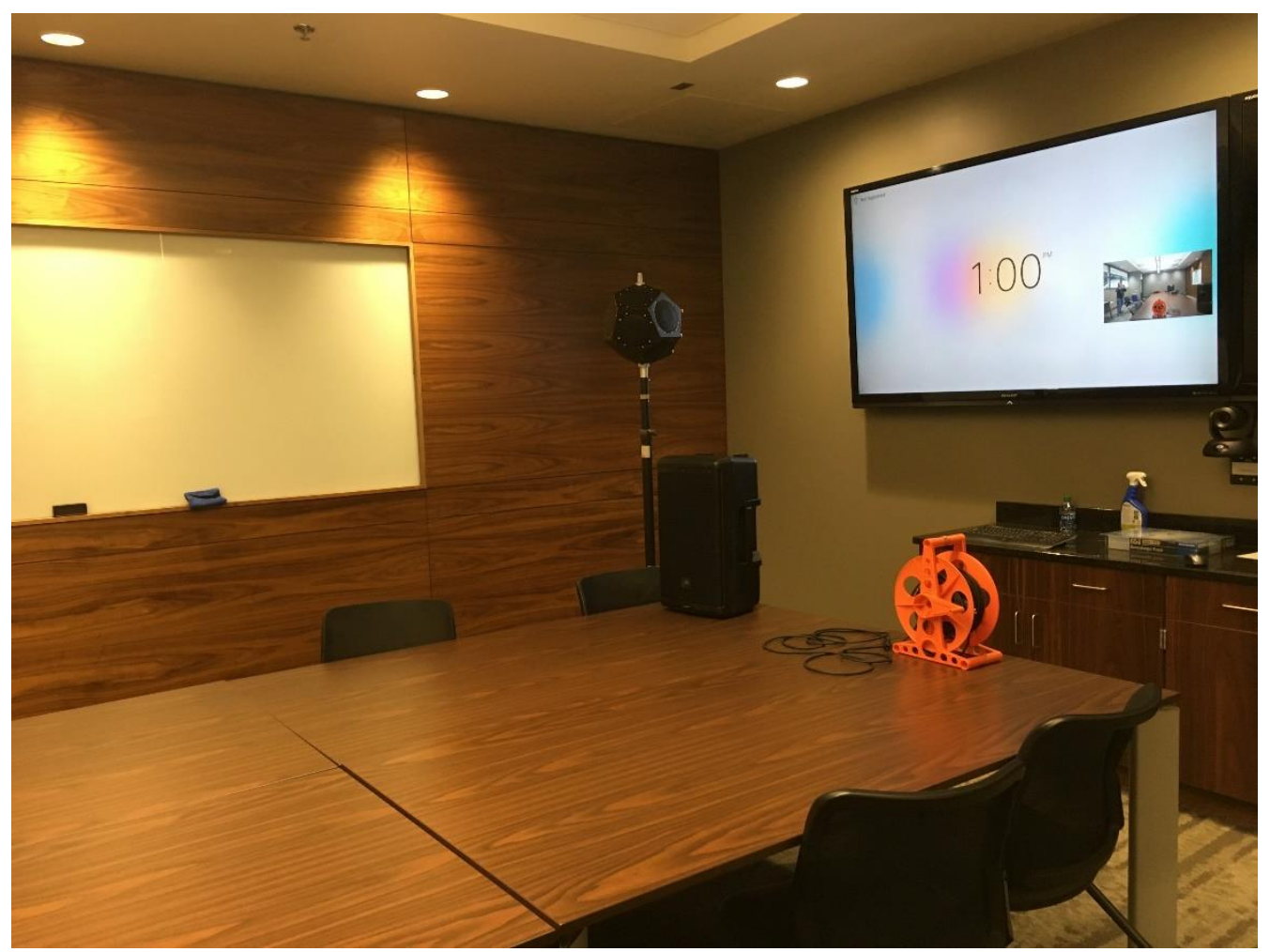

Figure 3.2: Photograph of the omnidirectional loudspeaker used for room impulse response measurements in a common talker position in Peter Kiewit Institute room 150 (a conference room); note that the other loudspeaker pictured was relevant to procedures used before the adoption of the convolution method (see Appendix B).

\subsubsection{Benchmarks and Expectations}

As stated earlier, the signals of interest to this study were impulsive noise (with an expected kurtosis level of 5 or more $\mathrm{dB}$ ), white noise (with an expected kurtosis level of $0 \mathrm{~dB}$ ), and a pure tone (with an expected kurtosis level of $-3 \mathrm{~dB}$ ). In terms of distributions, this study chose to use random noise following the Laplace distribution to model a continuous noise signal with impulsive character and a series of rectangular pulses lasting one-tenth of a second as a wholly impulsive signal; pure tones and white noise corresponded to the arcsine and Gaussian distributions respectively (as discussed in Chapter 2). The values of kurtosis for each distribution directly inform the expected kurtosis levels stated above, and are illustrated by the 
distributions in Figures 3.3-3.6 below. If these graphs were applied to quantities of interest to this thesis, the horizontal-axis label "Example $\mathrm{x}$ " for Figures 3.3-3.5 would be replaced by "Sound Pressure $(\mathrm{Pa})$ " and the horizontal axis itself might need to be rescaled to realistically approximate real sound pressures, while the vertical axis would correspond to the probability of a random pressure from the signal under examination being equal to a given horizontal-axis value. Figure 3.6 is slightly different in that it depicts a time-domain signal rather than a probability distribution; in this case the horizontal-axis label would read "Time (s)" and the vertical-axis label would read "Sound Pressure $(\mathrm{Pa})$ " (as well as potentially requiring rescaling to depict realistic numbers) if the graph were applied to the quantities under study. All four of the following figures are left as they are so that they may serve as a general visual supplement to the previously-discussed relationship between the kurtosis level metric and the concept of treating noise exposures as distributions of sound pressure that serves as a pillar of this study.

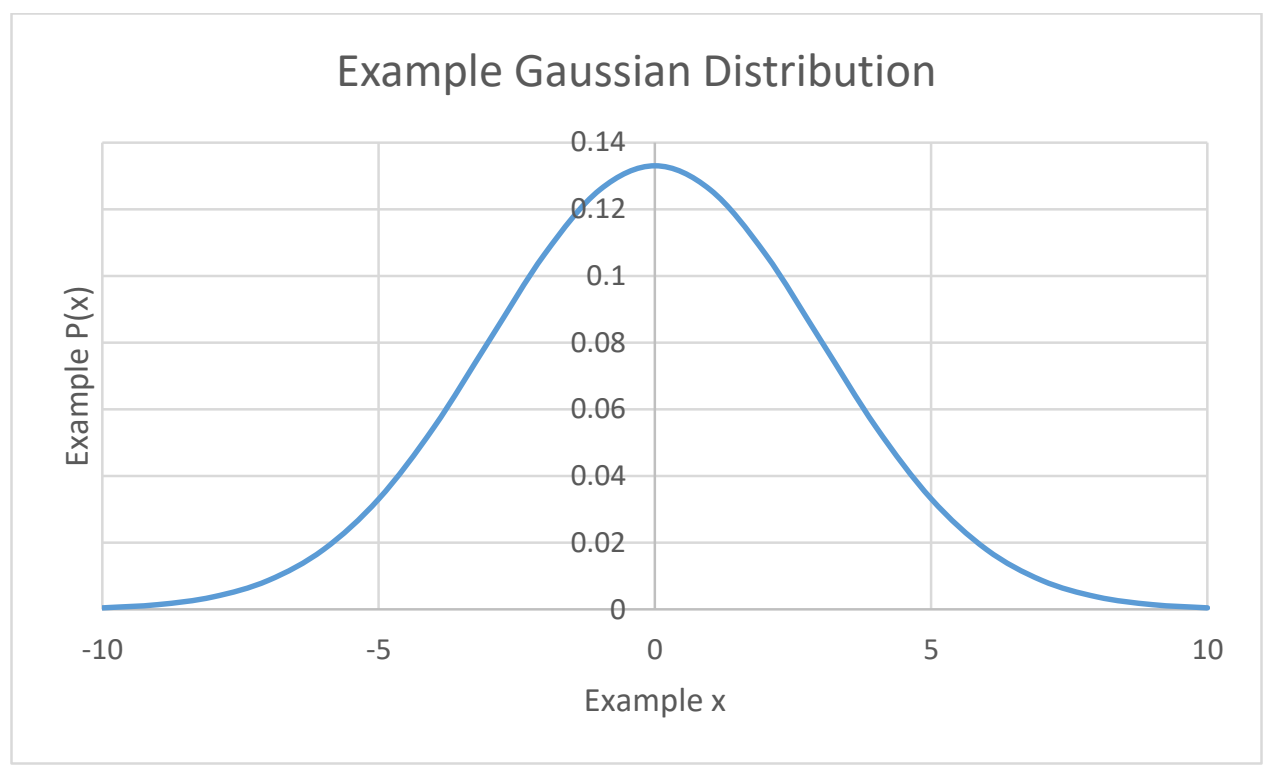

Figure 3.3: Example of a Gaussian distribution, created using Excel; this is the distribution that yields a kurtosis level of $0 \mathrm{~dB}$, leading to its association with white noise. 


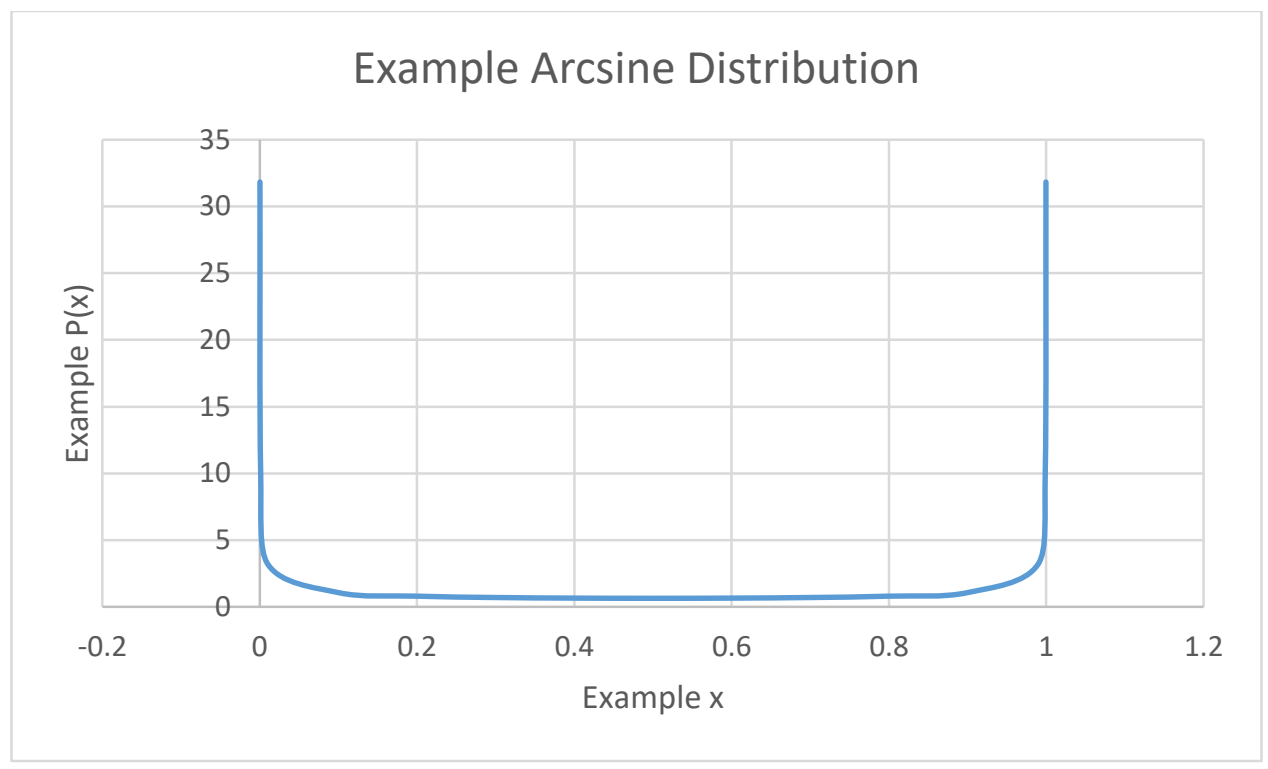

Figure 3.4: Example of an arcsine distribution, created using Excel (note that for purposes of this study, this distribution was considered bimodal with tails trending asymptotically towards zero because asymptotic behavior towards infinity is exceedingly rare in physical acoustical systems, regardless of the arcsine function's domain limits). This is the distribution that produces a kurtosis level of $-3 \mathrm{~dB}$, leading to its association with the pure tone.

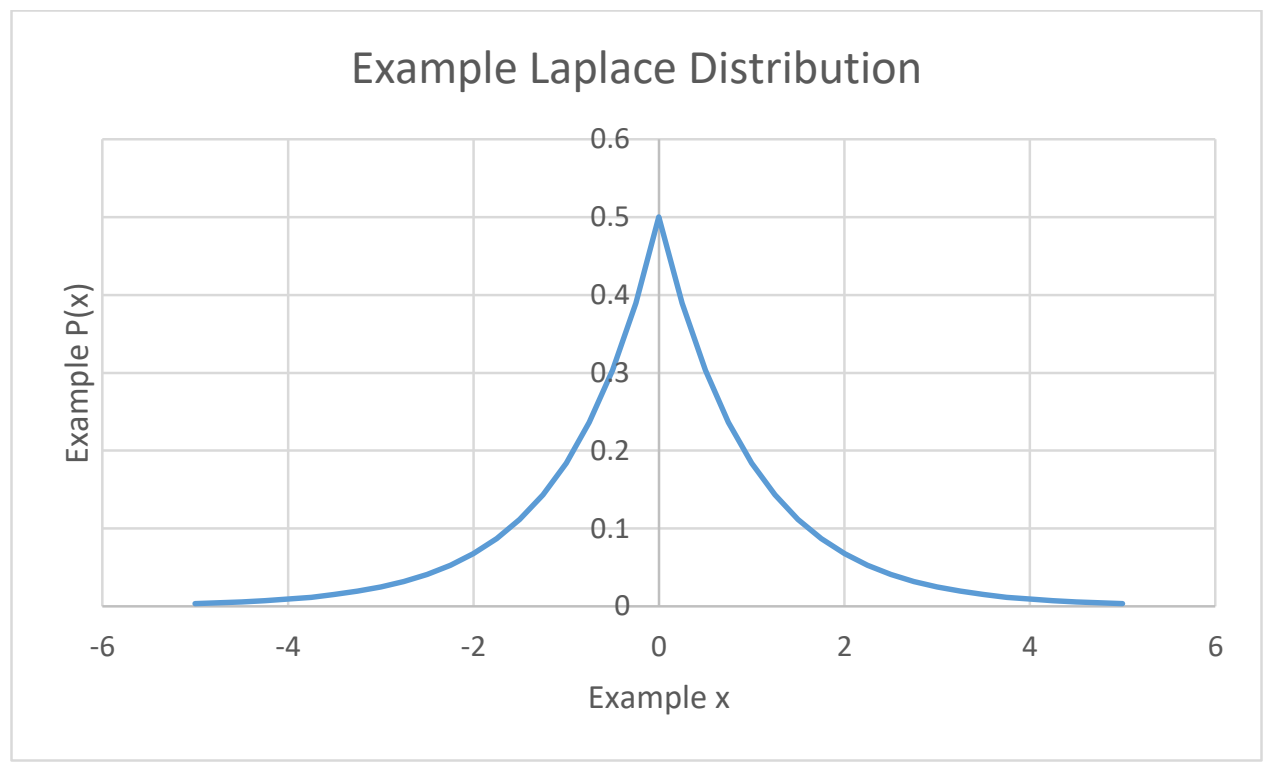

Figure 3.5: Example of a Laplace distribution, created using Excel; this is one of several distributions known in statistics to have a high kurtosis, and was selected to create a continuous noise signal with impulsive character for this study. 


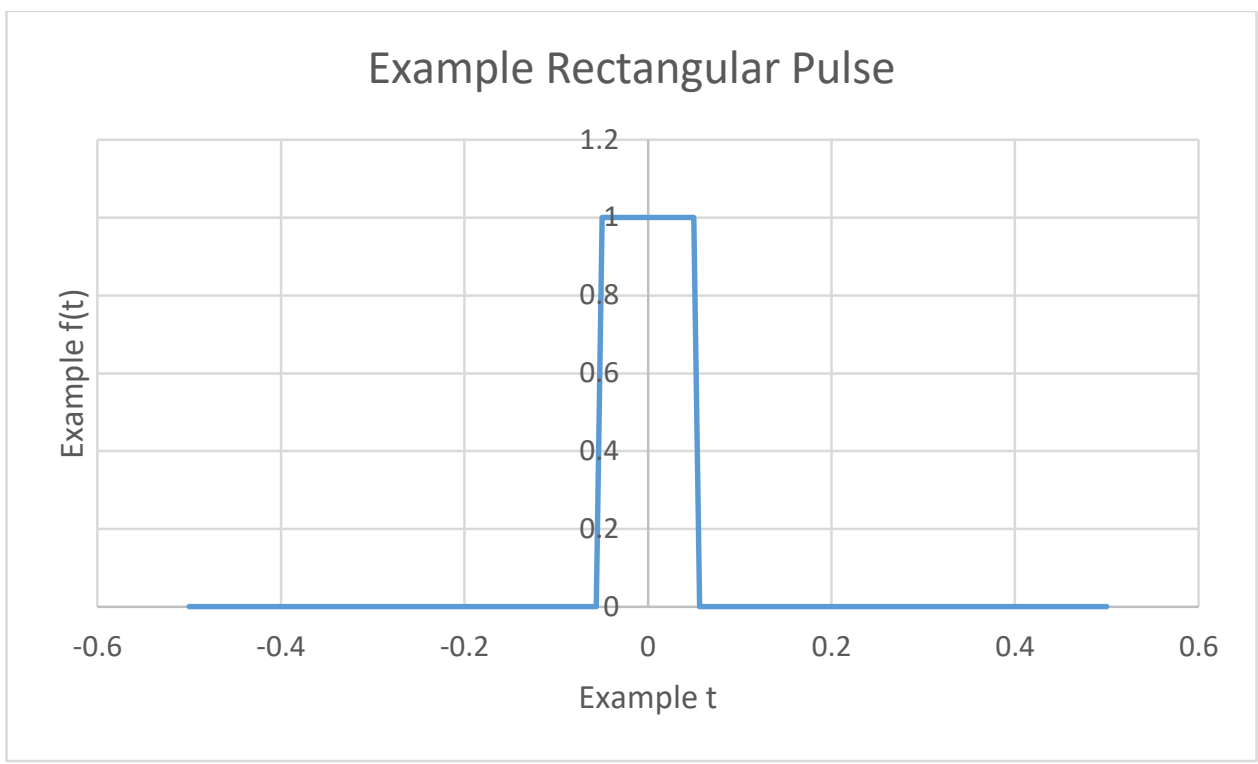

Figure 3.6: Example of a rectangular pulse in time, created using Excel; this is one approximation of an acoustical impulse if $f(t)$ is in units of pressure. Thus, it was used to create an impulsive noise signal for this study.

The impulse response of the anechoic chamber at Boys Town National Research

Hospital's east campus was put through the convolution procedure described in Section 3.1.1 to create reference kurtosis levels using the study's own methodology. This step was important to take because the signals used in this study were highly unlikely to be exactly like those analyzed by Zechmann. Ensuring that the study had an internal frame of reference to supplement the external one provided by Zechmann would create a failsafe against misinterpretation of the results' implications about Zechmann's predictions. These reference levels are presented by signal in Chapter 4, along with the data they contextualize.

Zechmann's predictions regarding the kurtosis level's behavior under room acoustical effects encompassed both absorption and scattering properties, with further distinctions made on the scattering side of the topic. First, he predicted that none of the signals' kurtosis levels would change due to room absorption, and that the kurtosis level of white noise would similarly not change under variation in room scattering. Second, he predicted that the kurtosis levels of pure tones and impulsive noise would shift towards the expected value of white noise with increased 
room scattering (Zechmann 2019). These predictions were used as a benchmark in evaluating how the data collected during this study answered the research question.

\subsection{Room Characteristics}

\subsubsection{Room Dimensions and Geometry}

Room volume and surface area must be known before any reverberation time equations can be used to calculate absorption coefficient. As alluded to in Section 3.1.1, the rooms' dimensions were measured in feet and inches, down to fractional inches thanks to the precision of the measurement tool; since the metric versions of the reverberation time equations were used in EASERA, the measured dimensions were rounded to the nearest inch before conversion into decimal meters for use in those equations, and in estimating the room's critical distance. The formula used for the latter calculation is as follows, where $r_{c}$ represents the critical distance in meters, $V$ represents the room volume in cubic meters, and $R T$ represents the room's reverberation time in seconds:

$$
r_{c}=0.057 \sqrt{\frac{V}{R T}}
$$

Further, since the rooms chosen for this study were mostly non-rectangular in shape, it would have been very difficult to automate volume and surface area calculation for them. Thus, as seen in the appendix (A-1), it was decided that volume and surface area should be calculated by hand and taken as inputs into the automated portion of the absorption coefficient calculation. In cases where ceiling height was significantly non-uniform but the ceiling was not sloped, such as room 158 in the Peter Kiewit Institute (a classroom), the volumes of areas with different heights were calculated separately and added together. In cases where the ceiling was sloped, the 
Pythagorean theorem was used to estimate the length of the sloped elements for purposes of surface area, and volume was estimated as the sum of rectangular and triangular prisms as appropriate. For irregular room shapes, a rectangle (or semicircle, or whatever shape with an easily calculable area approximated the room best) larger than the piece of room under consideration was used as a base, and appropriate areas of exclusion were subtracted (if necessary) to finalize the calculation. No computer modeling techniques were used in these processes, as the investment of time and computing power was deemed too great for the relatively low importance of room volume and surface area to the research question.

\subsubsection{Sound Absorption Coefficient}

One metric used in this study to quantify a room's acoustical characteristics was its sound absorption coefficient. This is a commonly analyzed metric in the field of architectural acoustics, and is well accepted as a representative metric for a room's sound absorption properties. For an example of an absorptive surface encountered during this study, see Figure 3.6. In this study, rooms' sound absorption coefficients were calculated from the room's reverberation time, which was obtained from EASERA. EASERA outputs both the T20 (an estimate of the reverberation time in which the time it would take for sound to decay by $60 \mathrm{~dB}$ is extrapolated from a measured $20 \mathrm{~dB}$ decay) and the $\mathrm{T} 30$ (a similar estimate of reverberation time in which the time of a $60-\mathrm{dB}$ decay is extrapolated from a measured $30 \mathrm{~dB}$ decay) approximations. 


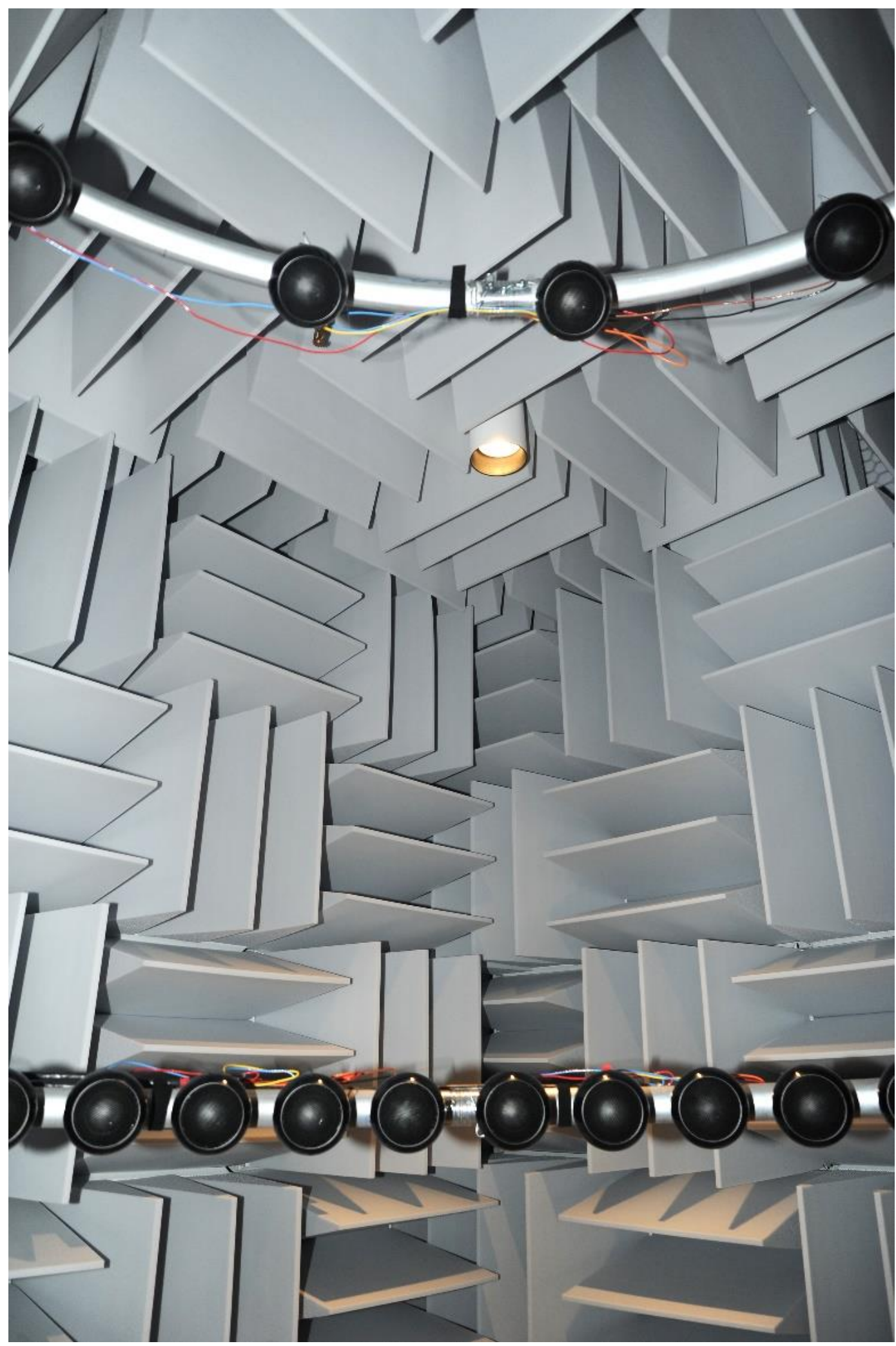

Figure 3.6: Example of strongly absorptive surfaces; photograph of the Boys Town National Research Hospital anechoic chamber courtesy of the BTNRH Marketing Department 
Which approximation of the reverberation time was selected for a given room depended on the room's background noise level, as certain levels of separation (generally equal to the number of decibels of decay being measured plus fifteen) between the initial point of the sound's decay and the background noise level are required to ensure that the estimate is maximally accurate (ISO 2008). The approximation that met these criteria (or which came closest to meeting them, in cases where the background noise level was too high for any to be met — see Section 4.1.1) was taken to be representative of the room, and the analysis proceeded by approximating the room's reverberation time as an average of its values for the octave bands from $250 \mathrm{~Hz}$ to $2000 \mathrm{~Hz}$, inclusive. From that point, self-written MATLAB code was used to first check the absorption coefficient using a rearrangement of the Sabine formula for reverberation time:

$$
R T=0.16 \frac{V}{\alpha S} \text {, when } \alpha<0.2 \text {, }
$$

where $V$ is the room volume in cubic meters, $\alpha$ is the room's sound absorption coefficient (dimensionless), and $S$ is the room's surface area in square meters. Room volume and surface area were calculated in accordance with the guidelines and simplifying assumptions detailed in Section 3.2.1. The calculation was then repeated using a rearranged form of the Norris-Eyring formula for reverberation time:

$$
R T=0.16 \frac{V}{S \ln (1-\alpha)}, \text { when } \alpha \geq 0.2 \text {, }
$$

where $V, \alpha$, and $S$ represent the same quantities as they do in the Sabine formula. It should be noted that both equations assume a diffuse sound field, which is not always true in general acoustical practice and was not always true in this study (further discussion of this assumption can be found in Section 4.1.1). 
The values obtained from those calculations were then compared to the equations' accepted regimes of validity (noted in the same equation lines used to present the respective formulae); if the absorption coefficients calculated from both formulae were within the acceptable range for one of them, then that formula's value was taken to be an accurate reflection of the room's real properties. In cases where the calculated absorption coefficients pointed to different formulae, the Norris-Eyring value was assumed to be more accurate due to its larger regime of validity. For information on which rooms' absorption coefficients were calculated using the Sabine formula and which rooms' absorption coefficients were calculated using the Norris-Eyring formula, see Table 4.1.

\subsubsection{Number of Peaks in the Impulse Response}

The predictions that this study set out to test are based in part on a room's sound scattering properties. Upon initial inquiry, the literature revealed little in the way of methods for directly calculating a room's total scattering capabilities in a field setting (Cox and D'Antonio 2004). However, in the course of that research, an alternative means of assessing test rooms' scattering properties became clear: room diffusion metrics. Those quantities assess how well a room scatters sound to achieve a diffuse sound field, or one where sound incidence on a receiver is equally likely from all directions (Long 2014). Thus, a room diffusion metric would serve as an acceptable substitute for a metric that directly addresses scattering. So, the search was widened to include such quantities. For an example of a surface that contributes to sound scattering and increased room diffusion which was encountered during the study, see Figure 3.7 below. 


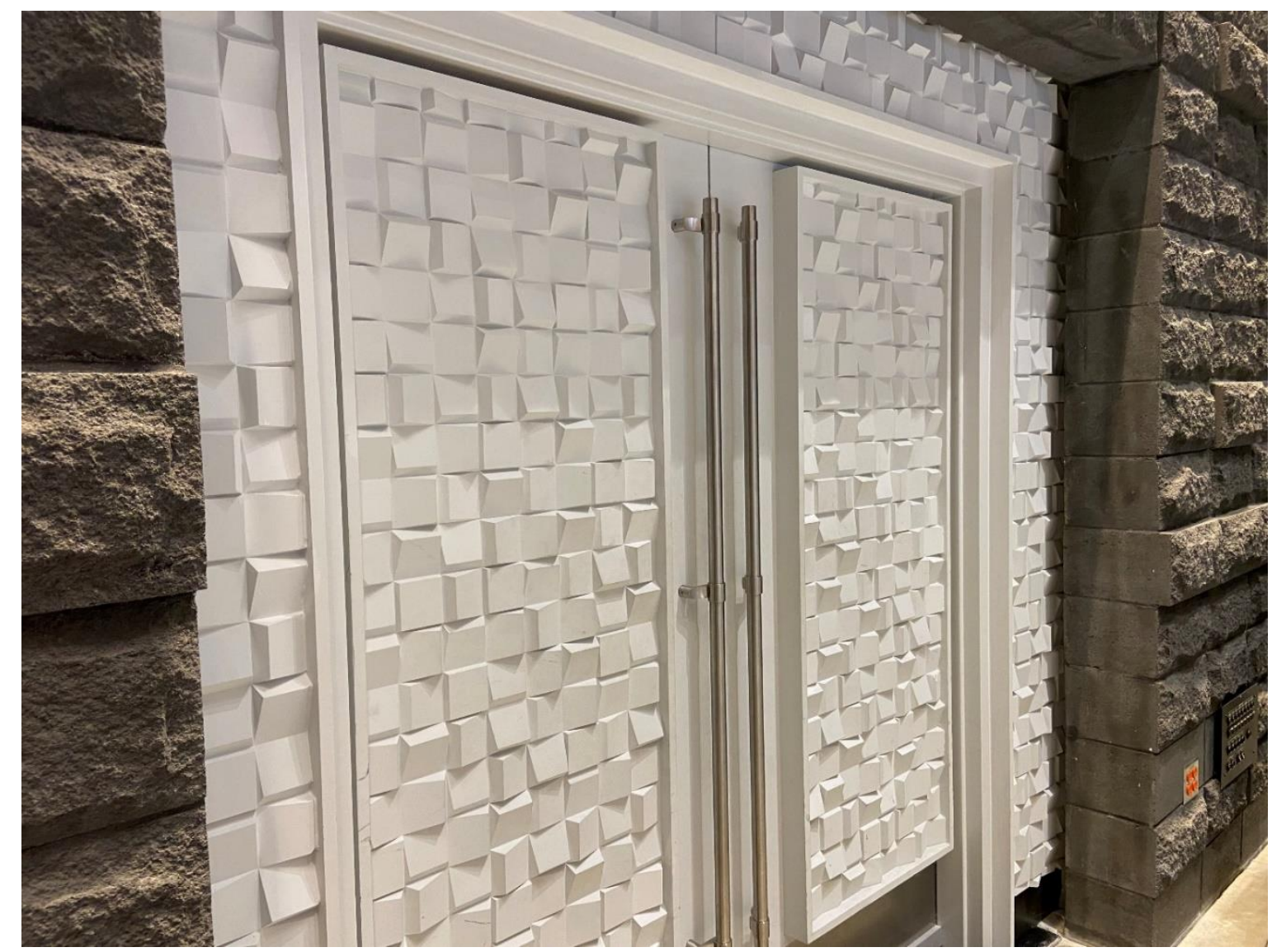

Figure 3.7: Example of a surface designed to augment a room's sound scattering properties; photograph taken in the Strauss Performing Arts Center recital hall

According to previous work by Bliefnick (2016), three main methods of assessing room diffusion were proposed in the early 2010s: transition time (Jeong, Brunskog, and Jacobsen 2013), degree of time series fluctuation (Hanyu 2014), and number of peaks in the impulse response (Jeon, Jang, and Kim 2013). Each had developed its own base of proponents and ideal use cases by the time of Bliefnick's research, which consisted of a statistical analysis of how effective each assessment method was in a controlled environment. Ultimately, it was determined that the number of peaks was the most effective of the trio. This study therefore adopted Bliefnick's outlook and chose to assess diffusion (and thus implicitly address scattering) through the number of peaks in the room's impulse response. 
As laid out in the paper which introduced it (Jeon, Jang, and Kim 2013) and in Bliefnick's analysis, the number of peaks in a room's impulse response assesses the fine structure of an impulse response's early stages, rather than depending on late stages. In Bliefnick's experience, this allowed the metric to more directly capture diffusive behavior without interference from or dependence on factors like wall absorption and source/receiver configuration (2016). There is no true calculation involved in determining this metric's value for a given room, as it relies on manual or automated counting of peaks above a given threshold. This threshold is determined, according to the authors who introduced the method, by using Morlet wavelets to precisely calculate the 20-dB-down point from the highest peak of the room impulse response. However, in both Bliefnick's assessment of the method and in the author's review of that paper, those authors did not present a detailed enough summary of their methodology for it to be replicated. Therefore, this study adopted Bliefnick's proposed alternative methodology, which was to transform the impulse response sound pressure data (part of EASERA's output) by taking their absolute values and converting them into decibels using MATLAB. From that point, the $-20 \mathrm{~dB}$ threshold suggested in the source paper was implemented and MATLAB counted the qualifying peaks (see Appendix A for the code in its entirety). Additionally, the source paper's authors recommended that the numbers of peaks be normalized by the number of peaks calculated for an anechoic chamber, to ensure that rooms of different sizes and geometries could be compared on even ground (Jeon, Jang, and Kim 2013). The anechoic impulse response discussed in Section 3.1.2 was used to find this normalization constant in this study. See Figure 3.8 for an example of the transformed data and the portion of the room impulse response under consideration when using this metric. A table containing each room's number of peaks can be found in Section 4.1.2 (Table 4.3). 


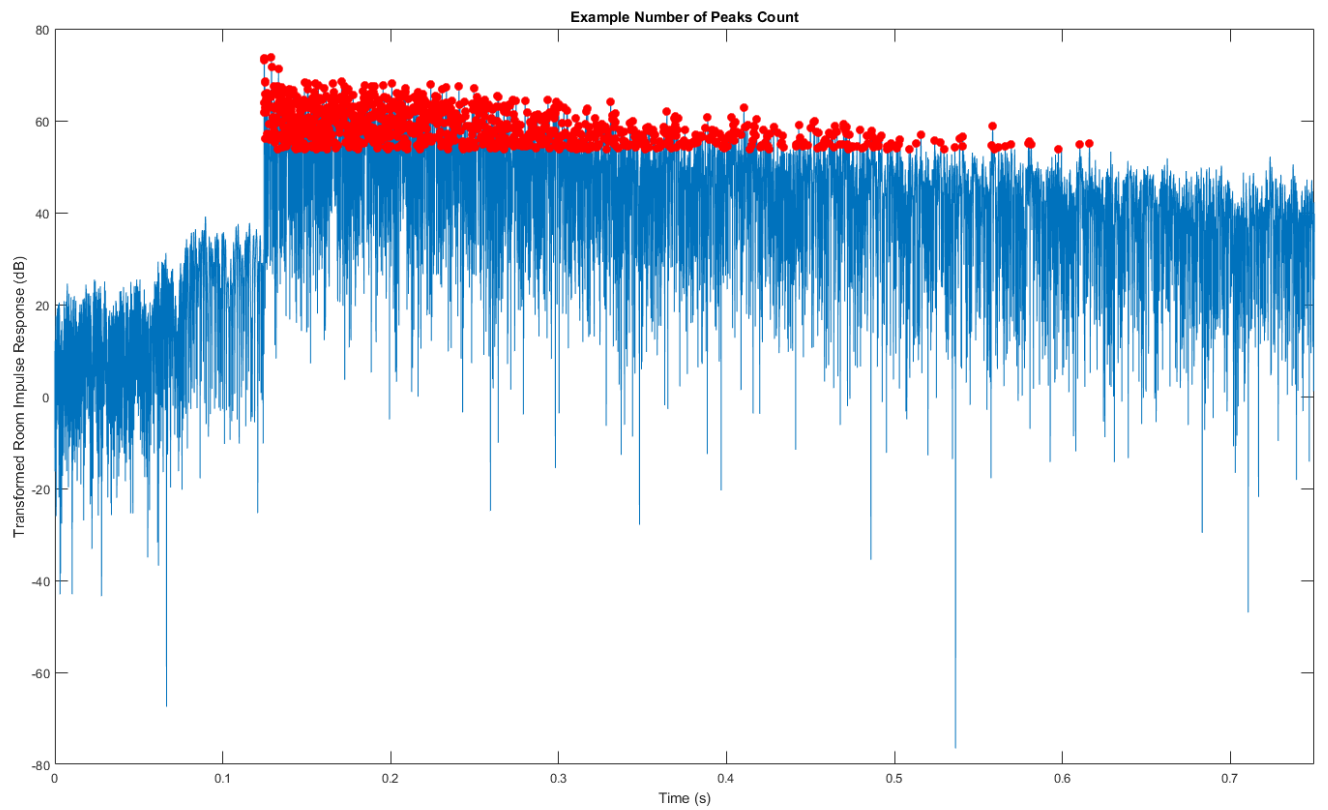

Figure 3.8: The early portion of the Strauss Performing Arts Center Recital Hall's impulse response, transformed using Bliefnick's methodology; peaks that counted towards the number of peaks metric are marked with red dots. 


\section{Chapter 4: Results}

\subsection{Room Properties and Signals of Interest}

Since the sound absorption and sound scattering properties of each test room, as well as the nature of each signal of interest, are fundamental to the study's results, they are presented separately here, along with a brief recapitulation of how they were dealt with and how their relationships were expected to turn out.

\subsubsection{Room Absorption Coefficients}

Sound absorption coefficient, as discussed in Section 3.2.1, is a common metric for the sound absorbing capabilities of a room; it was predicted that it would exert little to no influence on the kurtosis level metric. For purposes of this study, each room's absorption coefficient was calculated from its reverberation time; that metric in turn was calculated from measured impulse responses using EASERA. The data used in that process were collected from playing a set of eight sine sweeps into each room (plus one presend). After applying a best practice that states the separation between the test sound's highest level and the background noise level should be a number of decibels equal to the measured decay plus fifteen, the most appropriate approximation of the reverberation time (T20, T30, etc.) was determined. Finally, a self-written MATLAB program used the average reverberation time across the $250 \mathrm{~Hz}$ to $2 \mathrm{kHz}$ octave bands (inclusive) and rearranged forms of the Sabine and Norris-Eyring formulae for reverberation time to estimate the absorption coefficient of each room. The equations can be found in Section 3.2.1, and Table 4.1 presents the equation determined to be most valid for each room, as well as the value of absorption coefficient it produced. More detailed consideration of the topic of selecting 
equations and reverberation time approximations can be found in the aforementioned Section

\subsection{1.}

Table 4.1: Reverberation time decisions, absorption coefficients, and related information for each test room

\begin{tabular}{|c|c|c|c|c|c|c|c|}
\hline $\begin{array}{c}\text { Room } \\
\text { (purpose) }\end{array}$ & $\begin{array}{c}\text { Volume } \\
\left(\mathbf{m}^{\mathbf{3}}\right)\end{array}$ & $\begin{array}{c}\text { Approx. } \\
\text { Used }\end{array}$ & $\begin{array}{c}\text { Average } \\
\text { Reverberation } \\
\text { Time for 250 } \\
\text { Hz-2 kHz (s) }\end{array}$ & $\begin{array}{c}\text { Equation } \\
\text { Used }\end{array}$ & $\begin{array}{c}\boldsymbol{\alpha} \\
\text { (unitless) }\end{array}$ & $\begin{array}{c}\text { Background } \\
\text { Noise Level } \\
\text { (dBA) }\end{array}$ & $\begin{array}{c}\text { SNR } \\
\text { (dB) }\end{array}$ \\
\hline $\begin{array}{c}\text { Boys Town } \\
\text { National } \\
\text { Research } \\
\text { Hospital } \\
\text { AV Lab }\end{array}$ & 87.12 & T20 & 0.0015 & $\begin{array}{c}\text { Norris- } \\
\text { Eyring }\end{array}$ & 0.9995 & 32.2 & 42.7 \\
\hline $\begin{array}{l}\text { Peter Kiewit } \\
\text { Institute 150 } \\
\text { (conference) }\end{array}$ & 203.7 & T20 & 0.76 & $\begin{array}{c}\text { Norris- } \\
\text { Eyring* }\end{array}$ & 0.19 & 39.2 & 18.4 \\
\hline $\begin{array}{l}\text { Peter Kiewit } \\
\text { Institute 158 } \\
\text { (classroom) }\end{array}$ & $1,403.3$ & T20 & 1.04 & $\begin{array}{c}\text { Norris- } \\
\text { Eyring }\end{array}$ & 0.22 & 44.2 & 44.5 \\
\hline $\begin{array}{c}\text { Strauss } \\
\text { Performing } \\
\text { Arts Center } \\
\text { Recital Hall }\end{array}$ & $1,205.8$ & T20 & 1.92 & $\begin{array}{c}\text { Norris- } \\
\text { Eyring }\end{array}$ & 0.14 & 45.9 & 17.0 \\
\hline
\end{tabular}

*Note that the two formulas yielded results on opposite sides of the $\alpha=0.2$ dividing line established in Chapter 3, so it was assumed that the Norris-Eyring formula produced a better estimate.

NOTE: Background noise levels are listed as measured by the sound level meter directly; SNR values stated in this table are for the overall measurement and were provided by EASERA.

One detail that is important to present alongside these data is the fact that both the Sabine and Norris-Eyring equations assume diffuse sound field conditions within the room, which may have been less than perfectly true (as is the case with many rooms examined by acousticians). As a corollary to the assumption of diffuse conditions, it is also expected that sound absorption be uniformly distributed across the space's surface area for the forms of the Sabine and NorrisEyring formulae used in this study to be perfectly applicable. That was untrue in both rooms from the Peter Kiewit Institute, as well as in the Strauss Performing Arts Center recital hall. In each of the aforementioned rooms, the absorptive materials were concentrated on one or two 
surfaces, most notably in the form of carpet in the PKI rooms or the one set of curtains within the recital hall.

As a final note, the quality of measurements taken for this study varies (as exemplified by the SNR, or signal-to-noise ratio, column of Table 4.1). This is largely attributable to the fact that while the author was able to reserve specific rooms for data collection, the building and rooms around the test rooms were still in normal use. This created background noise issues, particularly in PKI 150 (whose background noise included contributions from repeated opening and closing of a nearby restroom door), that reduced the measurement equipment's ability to capture the room's performance accurately. Further potential causes for low SNR include insufficient amplification of the sine sweep for the volume of the space and the activation and deactivation of building systems during measurement procedures (which are both applicable to the case of the Strauss recital hall, the other room that produced a notably low SNR).

Continuing on the topic of room defects, it is important to note that any potential trends in kurtosis levels of pure tones due to room effects could be confounded by rooms' acoustical modes. Room modes are dominant in low frequency ranges, and are influenced by room geometry. Each room's unique transition point between modal dominance and more ideal diffuse conditions is described by its characteristic Schroeder frequency (Long 2014). To ensure as much as possible that each room's data were not impacted by such defects, its Schroeder frequency was calculated after the room volume $V$ and reverberation time $R T$ were available to use in Equation 6 below:

$$
f_{S}=2000 \sqrt{\frac{R T}{V}} .
$$


The pure tone used in this study was a sine wave at $1 \mathrm{kHz}$; thus, it exceeded each room's Schroeder frequency by a significant margin (see Table 4.2), reducing worries that any modal behavior in the measured impulse response would cause problems during the convolution phase of analyzing pure tones' behavior.

Table 4.2: Schroeder frequencies of all test rooms

\begin{tabular}{|c|c|}
\hline Room (purpose) & Schroeder Frequency (Hz) \\
\hline $\begin{array}{c}\text { Boys Town National Research Hospital AV } \\
\text { Lab }\end{array}$ & 21.4 \\
\hline Peter Kiewit Institute 150 (conference) & 92.7 \\
\hline Peter Kiewit Institute 158 (classroom) & 48.5 \\
\hline Strauss Performing Arts Center Recital Hall & 63.1 \\
\hline
\end{tabular}

\subsubsection{Numbers of Peaks in Room Impulse Responses}

The number of peaks in a room's impulse response is a proposed metric for assessing a room's sound scattering properties in a field setting; the process that went into selecting this particular metric for the study is detailed in Section 3.2.3. Essentially, the metric entails counting the number of peaks between the highest sound pressure level in the impulse response and the 20-dB-down point from that level. Table 4.3 presents the number of peaks for each room tested in this study, which were counted automatically by MATLAB after impulse response data were imported from EASERA. The values presented in Table 4.3 are reasonable when compared to the values Bliefnick calculated for rooms without time-filtering the impulse response data (which ranged from approximately 400 to 800 in a room of volume 1104 cubic meters, less than the volume of the recital hall tested in this study by about 100 cubic meters) (Bliefnick 2016). A higher number of peaks corresponds to a more diffuse room (and therefore implies greater sound scattering capabilities on the room's part). In terms of room acoustical rules of thumb, these results are still sensible; it is conventional wisdom for acoustical design that classrooms and 
conference rooms have similar properties and recital halls be less absorptive and better at creating diffuse sound fields than rooms used for speech.

Table 4.3: Normalized number of peaks in each room's impulse response

\begin{tabular}{|c|c|}
\hline Room (purpose) & Number of Peaks \\
\hline $\begin{array}{c}\text { Boys Town National Research Hospital AV } \\
\text { Lab }\end{array}$ & 1 \\
\hline Peter Kiewit Institute 158 (classroom) & 318 \\
\hline Peter Kiewit Institute 150 (conference) & 826 \\
\hline Strauss Performing Arts Center Recital Hall & 1068 \\
\hline
\end{tabular}

\subsubsection{Signals of Interest and Predictions about their Behavior}

Four signals of interest were selected for this study. Three of them came from the studies that directly inspired the author to undertake this research: the pure tone, white noise, and impulses. These three signals were expected, per Zechmann (2019), to have kurtosis levels of -3 $\mathrm{dB}, 0 \mathrm{~dB}$, and 5 or more $\mathrm{dB}$ respectively under anechoic conditions. The fourth signal was added by the author to approximate complex noise exposures that are continuous and have impulsive components; it was generated by transforming uniformly distributed random numbers into random numbers following the high-kurtosis Laplace distribution. There was no preexisting expectation for this signal's kurtosis level, but common sense would suggest that it fall between 0 and $5 \mathrm{~dB}$, as it contains impulsive components moderated by continuous components. This intuition was affirmed once kurtosis level calculations began, as can be seen in Figure 4.1.

These signals were computer-generated within MATLAB as part of the same program that convolved the signals with room impulse responses and calculated the kurtosis levels of the room-specific convolution output. As such, the author had the option to create them to arbitrary specifications; the finalized format for these signals was to match EASERA's sampling rate of $44.1 \mathrm{kHz}$ to a duration (translated from MATLAB array length) of 5 minutes (300 seconds). The 
former part of the decision was made simply to ensure consistency between the different analysis tools used in the study, but the reasoning behind the latter part was more complex.

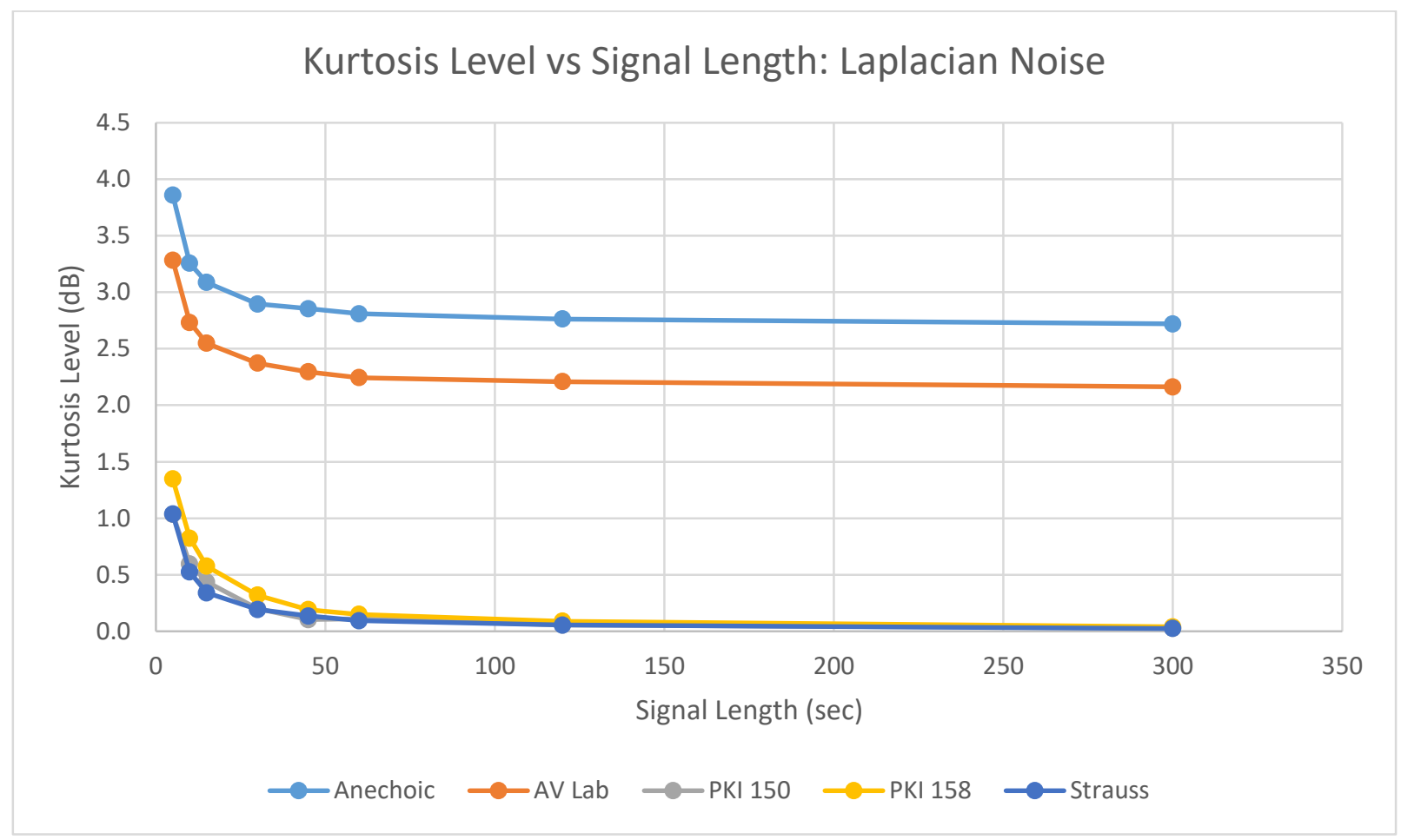

Figure 4.1: Graphic showing the changes in kurtosis level of Laplacian noise as the duration of the computergenerated signal increases, including the anechoic data used as a reference. Similar asymptotic behavior is observed in the other four signals of interest.

As evidenced by Figure 4.1, the longer the computer-generated signal was, the more its kurtosis level asymptotically approached a room-specific value. Given that the kurtosis statistic measures how likely it is that a random number from the distribution to which it belongs will be an outlier, the author decided to interpret this behavior as follows: shorter simulated noise exposures provide little enough information about their associated sound pressure distribution that more values would be identified as outliers than would be if the "exposure" were longer. Under this interpretation, the longest time-efficient signal possible was found to be 300 seconds long and assumed to be the most accurate rendering of the signals' associated sound pressure distributions. For signals of that length, the anechoic reference values for each signal were as 
follows: $-2.989 \mathrm{~dB}$ for the pure tone, $0.0251 \mathrm{~dB}$ for white noise, $2.719 \mathrm{~dB}$ for Laplacian noise, and $9.100 \mathrm{~dB}$ for the impulse series. These values are well within acceptable variation for different signal generation methods, especially considering that fractional decibels are indistinguishable differences to human perception. The only possible exception is the prodigious benchmark set for the impulse series, but that signal being more impulsive than expected is relatively unproblematic for this study.

Figure 4.1 can also be seen as evidence that room acoustical properties do affect the kurtosis levels of certain signals. While the statistical rigor behind this assertion is discussed in the following sections, it is worth noting that the higher kurtosis levels in the graph belong to the rooms with the lowest numbers of peaks in their impulse responses, and that the lowest kurtosis levels on the graph belong to the rooms with the highest numbers of peaks in their impulse responses. This could be a point in favor of Zechmann's prediction that noises with impulsive components would see their kurtosis levels decrease with increasing room scattering. To summarize the remainder of Zechmann's predictions before presenting the study's results, it was expected that no signal's kurtosis level would change with room sound absorption and that pure tones would see their kurtosis levels increase with increasing room scattering. White noise's kurtosis level was predicted to be unaffected by increasing room scattering.

\subsection{Kurtosis Levels with respect to Absorption Coefficient}

Figure 4.2 presents the data points and best fit lines for all four signals' kurtosis levels, plotted against absorption coefficient. Each signal type's data set was analyzed separately for purposes of quantifying (via correlation coefficient $r$ ) and evaluating (via a two-tailed $t$ test at $p<$ 
0.05, a common benchmark) the trend it potentially represents. For pure tones, the correlation coefficient was calculated to be -0.487 , indicating a negative relationship of moderate strength. The associated two-tailed p-value was 0.00058 , which indicates potential significance under the standards stated above. For white noise, the correlation coefficient was found to be 0.0224 , indicating a very weak positive relationship; the associated two-tailed p-value was 0.17 , which does not indicate any significance in this trend. For Laplacian noise, the correlation coefficient was calculated to be 0.997 , indicating a very strong positive relationship. However, the associated two-tailed p-value of 0.63 did not signal significance. Lastly, for the impulse series, the correlation coefficient was found to be 0.975 , indicating a very strong positive relationship; the associated two-tailed p-value was 0.013 , indicating potential significance. These results are presented more succinctly in Table 4.4 .

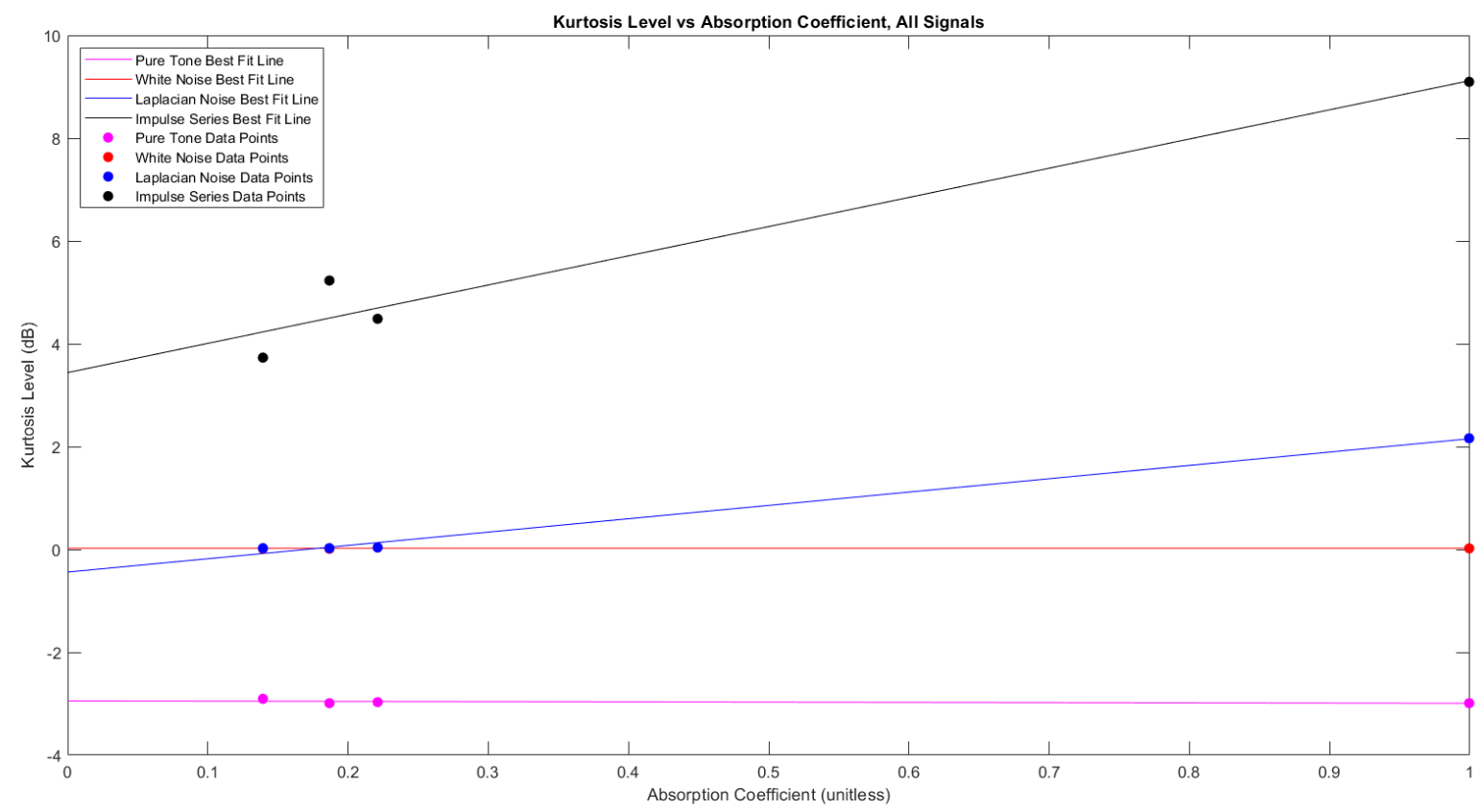

Figure 4.2: Kurtosis levels of all signals of interest (dB) plotted against absorption coefficient (unitless). Data points from left to right refer to the Strauss Recital Hall, PKI 150, PKI 158, and the BTNRH AV Lab. 
Of the four signals of interest, only two appear (visually) to fall in line with Zechmann's prediction that room sound absorption would not affect the kurtosis level metric: the pure tone and white noise. The pure tone's negative relationship appears to have some strength, but its scale is so small (tenths and hundredths of decibels) that it essentially behaves as expected. Both signals with impulsive components had positive trends instead of exhibiting the expected "neutral" behavior, and one of those relationships even has the appearance of statistical significance. This deviation from expectation merits further inquiry, especially since the low number of rooms tested in this study casts doubt on the reliability of these statistics.

\subsection{Kurtosis Levels with respect to Number of Peaks}

Figure 4.3 presents the data points and best fit lines for all four signals' kurtosis levels, plotted against the number of peaks in each room's impulse response. Each signal type's data set was analyzed separately for purposes of quantifying (via correlation coefficient $r$ ) and evaluating (via a two-tailed $t$ test at $p<0.05$, as before) the nature and significance (respectively) of the trend it potentially represents. For pure tones, the correlation coefficient was calculated to be 0.671, indicating a positive relationship of moderate-to-high strength. The associated two-tailed p-value was 0.10 , which does not imply any potential significance under the standards stated above. For white noise, the correlation coefficient was found to be -0.570 , indicating a moderately strong negative relationship; the associated two-tailed p-value was 0.11 , which does not indicate any significance in this trend. For Laplacian noise, the correlation coefficient was calculated to be -0.766 , representing a strong negative relationship. However, the associated two-tailed p-value of 0.11 did not signal significance. Lastly, for the impulse series, the correlation coefficient was found to be -0.790 , indicating a strong negative relationship; the 
associated two-tailed p-value was 0.11 , which does not belie potential significance. These results are presented more succinctly in Table 4.5 .

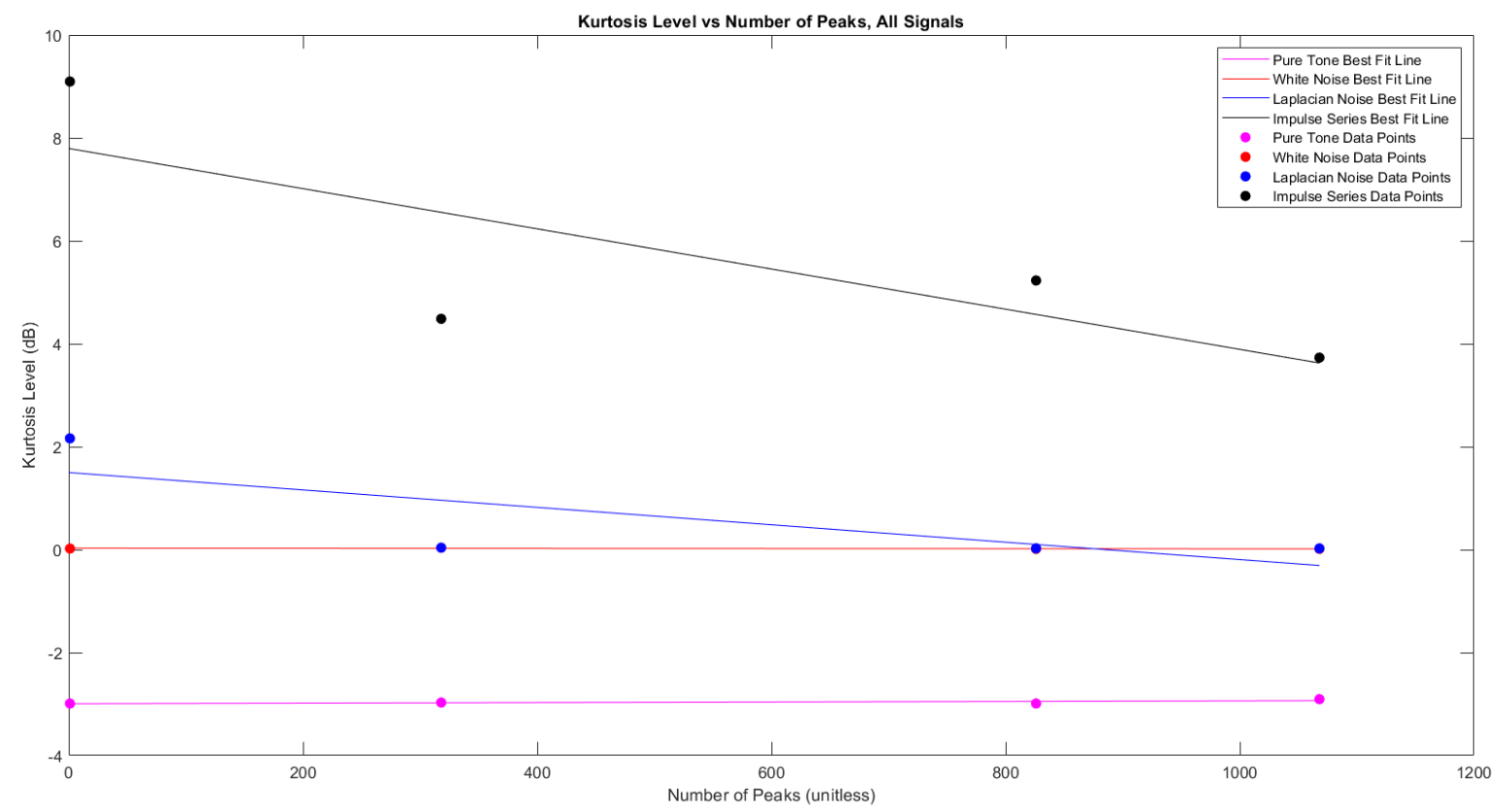

Figure 4.3: Kurtosis levels of all signals of interest $(\mathrm{dB})$ plotted against number of peaks (unitless). Data points from left to right refer to the BTNRH AV Lab, PKI 158, PKI 150, and the Strauss Recital Hall.

Of the four signals of interest, three appear (visually) to fall in line with Zechmann's prediction that as room sound scattering increases, the kurtosis levels of impulsive noises and pure tones would approach that of white noise (whose own kurtosis level would remain unchanged): the two signals with impulsive components and white noise. The pure tone's positive relationship appears strong, but its scale is so small (tenths and hundredths of decibels) that it essentially exhibits "neutral" behavior instead of increasing towards $0 \mathrm{~dB}$ in the highscattering regime. Both signals with impulsive components decreased towards $0 \mathrm{~dB}$ in that same regime as expected, and white noise exhibited a negative trend but essentially behaves "neutrally" due to the numbers that compose the trend varying on the same scale as the relationship observed for pure tones. Both the expected and unexpected behaviors merit further 
investigation, especially as their p-values uniformly come relatively close to significance despite the low number of data points available.

\subsection{Summary of Results}

This section summarizes the study's analytical outcomes. To briefly reiterate, no statistically significant results were found without caveats, especially regarding the low number of data points. This is an unfortunate consequence of the data collection process being interrupted by COVID-19 lockdowns. Nevertheless, any p-values that would indicate statistically significant results are colored red and italicized. This information is organized as follows: Table 4.4 presents the results pertaining to absorption coefficient, and Table 4.5 presents the results pertaining to number of peaks in the impulse response. In each table, the signals are presented in the same order in which they were discussed in Sections 4.2 and 4.3.

Table 4.4: Statistical summary of kurtosis levels with respect to absorption coefficient

\begin{tabular}{|c|c|c|c|c|}
\hline Sound Type & $\begin{array}{c}\text { Mean } \\
\text { Kurtosis } \\
\text { Level (dB) }\end{array}$ & $\begin{array}{c}\text { Standard } \\
\text { Deviation of } \\
\text { Kurtosis } \\
\text { Levels (dB) }\end{array}$ & $\begin{array}{c}\text { Correlation } \\
\text { Coefficient }\end{array}$ & p-value \\
\hline Pure Tone & -2.96 & 0.0402 & -0.487 & 0.00058 \\
\hline White Noise & 0.0223 & 0.0107 & 0.0224 & 0.173 \\
\hline Laplacian Noise & 0.563 & 1.07 & 0.997 & 0.629 \\
\hline Impulse Series & 5.64 & 2.39 & 0.975 & 0.013 \\
\hline
\end{tabular}

Table 4.5: Statistical summary of kurtosis levels with respect to number of peaks

\begin{tabular}{|c|c|c|c|c|}
\hline Sound Type & $\begin{array}{c}\text { Mean } \\
\text { Kurtosis } \\
\text { Level (dB) }\end{array}$ & $\begin{array}{c}\text { Standard } \\
\text { Deviation of } \\
\text { Kurtosis } \\
\text { Levels (dB) }\end{array}$ & $\begin{array}{c}\text { Correlation } \\
\text { Coefficient }\end{array}$ & p-value \\
\hline Pure Tone & -2.96 & 0.0402 & 0.671 & 0.105 \\
\hline White Noise & 0.0223 & 0.0107 & -0.570 & 0.106 \\
\hline Laplacian Noise & 0.563 & 1.07 & -0.766 & 0.106 \\
\hline Impulse Series & 5.64 & 2.39 & -0.790 & 0.109 \\
\hline
\end{tabular}


This study made some assumptions about the statistical nature of the data during the analysis process, which are worth making known at this stage. First and foremost is that the chosen statistical and analytical methods were applicable to the kurtosis level (which was treated as a quantity obeying a normal distribution itself by the nature of said methods). This may not necessarily have been the case, as several physical factors (which were probably interrelated, as discussed below) could have been influencing its behavior and therefore required a more nuanced approach. A more rigorous statistical treatment of the data would benefit the results' clarity, reliability, and validity, but this assumption was made so that analysis could progress at a respectable pace and so that the same easily comprehensible statistical analysis could be conducted on all parts of the data. Even if these methods were the correct choice, not enough data points were present to draw even somewhat definitive conclusions about the phenomena under study; that is another area in which future research can build on these findings.

An additional assumption made for the analysis portion of this project was that absorption and scattering were independent of each other and could therefore be analyzed separately. This assumption was deemed reasonable based on the experientially informed belief that the quantities of absorption added by a typical sound diffuser, and the quantities of sound scattering added by a typical sound absorber, are small in practical applications. However, data exist that countermand this belief, meaning that a more thorough look at this relationship is warranted in future studies so that its impact can be acknowledged in any standards, guidelines, or best practices that emerge from this body of research. 


\section{Chapter 5: Discussion}

\subsection{Implications of the Results}

The results presented in Chapter 4 may carry implications about the behavior of the kurtosis level metric under changes in room acoustical properties; this section is dedicated to discussing those potential implications. Two of the four signals of interest behaved in a way that aligned with Zechmann's predictions of room sound absorption exerting no effect on any signal's kurtosis level; those signals were white noise and the pure tone. The two signals with impulsive components saw their kurtosis levels increase with increasing room absorption. Two of these behaviors were marked as potentially significant by statistical testing: that of the pure tone and that of the impulse series. On the scattering side of the discussion, three of the four signals of interest behaved as predicted: white noise's kurtosis level remained essentially static with increasing scattering and the kurtosis levels of the signals with impulsive character decreased towards $0 \mathrm{~dB}$ as room scattering became stronger. The pure tone, on the other hand, essentially behaved "neutrally" instead of increasing towards $0 \mathrm{~dB}$ as expected. None of these trends returned statistically significant correlations. The combination of how few rooms contributed data and the lack of clear support from further statistical exploration of the data implies that this study's results provide insufficient information to reject or affirm Zechmann's predictions. Thus, future research could find that statistical analysis of these phenomena does not disprove the predictions, but this study cannot do so with the results it has. Nevertheless, the results are intriguing and could be informative when contextualized with the body of knowledge on impulsive noise. 
Lastly, it is worth reiterating a point about the kurtosis level that was alluded to in the preceding chapters. The kurtosis level is easily affected by small changes to the measured numbers contributing to its calculation, as demonstrated in multiple phases of the development of the data collection process. First, the number of samples of the impulse response fed into convolution can change the resultant kurtosis level even if all other variables are held constant; before the final "tail minimization" protocol was finalized, changing the impulse response length from 1.5 seconds to 5.9 seconds was enough to change the post-convolution kurtosis levels of the impulse series by as much as two decibels. Later, as discussed in Section 4.1.3, the length of the signal of interest itself was also discovered to have an impact on the resultant kurtosis level. These discussions do not even take into account the difficulties of measuring the effects this study was concerned with in a physical setting, where other background noises and equipment limitations can affect the data obtained. Since the kurtosis level as a metric is so procedurally sensitive, it is imperative that the collection and processing of data that will be used to report kurtosis levels be standardized. This observation has also been made by other researchers, such as Smalt et al (2017).

\subsection{Future Work}

Future research can complement, validate, and extend the work done by this study. This section details some areas identified by the author as having a potentially greater weight with respect to this study's achievements and shortcomings.

\subsubsection{Enhancing Rigor}

The most obvious extension of this study is to broaden the range of absorption and scattering under consideration. To restate information found in Chapter 4 , the usable data 
covered a range of absorption coefficients from 0.14-1.0 (unitless) and a range of numbers of peaks from 1-1068 (also unitless). The absorption coefficient can theoretically range from zero to one, so this study nominally considered the vast majority of the possible range. The possible range of number of peaks is less immediately clear, but this study's assessment of rooms' scattering properties is nevertheless subject to the same problem as this study's treatment of absorption coefficient: low $n$.

Considering more data points, and possibly a wider range of values of a scattering metric, could take several forms, but the most obvious would be to control for room volume and vary the absorption and scattering of the test space (probably a laboratory room or similar setting) through the addition and removal of acoustical treatment products. That approach was considered for this study, but was rejected due to the time and budget constraints under which the study was conducted (as discussed in Section 3.1.1). Alternatively, a room model created using software like CATT or ODEON could be used to achieve similar or better levels of control, with the added potential of idealizing the distribution of absorptive surfaces to better justify use of the Sabine and Norris-Eyring formulae. Ultimately, increasing the number of data points will increase the likelihood that the results of such a study accurately reflect the system's behavior and the confidence the research community can have in the conclusions drawn.

As discussed throughout Chapter 3, in Section 4.1.1, and in Section 4.4, several assumptions were made during the course of this study, on various scales. While no assumption was made without justification, as discussed in the initial treatment of each assumption, it is plainly obvious that substituting greater rigor for any and/or all of those assumptions would yield results with a greater degree of confidence behind them. For that reason, this avenue for validating the results of this study is suggested. Precise calculations of room surface area and 
volume, like those that would be produced by a computer model, for example, would increase the accuracy of the calculated absorption coefficient, which might lend additional clarity to the correlation between room sound absorption and the kurtosis level metric.

Of special note on this topic are the analytical assumptions outlined in Section 4.4. While analysis of variance and single-variable statistical calculations like least squares linear correlation and p-value significance testing are easily comprehensible, they may not be the best match for the situations and phenomena examined in this study. A researcher or research group with more time, computing power, and other applicable resources would be able to bypass the assumption that sound absorption and sound scattering properties of rooms are independent and potentially find a mathematical approach that aligns better with the phenomena under study. This would be an important step in validating and making actionable this study's results.

\subsubsection{Connections with Other Disciplines}

This study, while it stands at an intersection of noise control and architectural acoustics, does not take into account several other disciplines that are relevant to the topic of impulsive noise. Thus, complementary studies that either consider or originate from those disciplines will also make valuable contributions to the body of knowledge. Researchers who specialize in physiological and psychoacoustics, for example, might be able to build on previously conducted studies of impulsive noise's impact on people's bodily and mental/emotional health by leveraging the kurtosis level metric. The psychoacoustical effects of impulsive noise would also fit in well with the current blending of architectural acoustics and psychoacoustics into the new standard for soundscape design and analysis, ISO 12913 parts 1, 2, and 3 (ISO 2019). As another example, a specialist in underwater acoustics might be able to determine how impulsive noise translates to underwater settings (thereby getting at important topics like how it affects 
underwater ecosystems and structures). These and other intersections of acoustical disciplines (as applied to the topic at hand) would constitute complementary work that stands to push the field forward and create a stronger basis for informed decisions about the regulation of impulsive noise exposure and the mitigation of its associated risks.

\subsection{Summary}

This study was motivated by a gap in the body of knowledge regarding the effects of room acoustics on the kurtosis levels of various noise signals; this knowledge would bring better portrayals of the risk of hearing loss due to impulsive noise closer to being actionable by organizations that issue noise protection guidelines, such as the National Institute for Occupational Safety and Health in the United States. Research done before this point had established the kurtosis level as a viable metric for the additional pernicious effects of impulsive noise when combined with the A-weighted equivalent sound pressure level, as well as found benchmark kurtosis levels for some common types of noise. This study aimed to test predictions about the impact of room sound absorption properties and room sound scattering properties on the kurtosis levels of those specific sounds.

To test those predictions, a measurement protocol was developed that involved collecting both the room impulse response and background noise levels for a variety of rooms. Those room impulse responses were convolved with the signals of interest (a pure tone following an arcsine distribution, white noise following a Gaussian distribution, a complex noise following a Laplace distribution, and a series of impulses). The convolved waveforms were analyzed for their kurtosis, and room-specific kurtosis levels were calculated. These values were checked for correlation and statistical significance against each room's sound absorption coefficient and the 
number of peaks in its impulse response. While no definitive conclusions can be drawn due to the low number of rooms that contributed usable data, the author believes it entirely possible that predictions regarding absorption's lack of effect and scattering's signal-dependent influence on the kurtosis level could be accepted as true with future research. 


\section{References}

Atherley, G. R., and A. M. Martin. 1967. "Equivalent Continuous Noise Level as a Measure of Injury from Impact and Impulse Noise." Annals of Occupational Hygiene 14 (11): 389 99.

Berger, Elliott H., Rick Neitzel, and Cynthia A. Kladden. 2015. "Noise Navigator Sound Level Database." 3M Occupational Safety \& Environmental Health Division.

Bliefnick, Jay Michael. 2016. "Investigation of Subjective Perception \& Objective Metrics of Acoustic Room Diffusion.” University of Nebraska - Lincoln.

Broch, Jens Trampe. 1980. "Amplitude Distributions of Industrial Noises and Their Possible Effects on Hearing Damage." In Proceedings of Internoise 1980, 963-66. Miami, FL.

Coles, R Ross A, Georges R Garinther, David C Hodge, and Christopher G Rice. 1967. "Hazardous Exposure to Impulse Noise." Journal of the Acoustical Society of America 43 (2): 336-43.

Cox, Trevor J., and Peter D'Antonio. 2004. Acoustic Absorbers and Diffusers: Theory, Design, and Application. 1st ed. New York, NY: Spon Press.

Davis, Robert I., Wei Qiu, Nicholas J. Heyer, Yiming Zhao, MS Qiuling Yang, Nan Li, Liyuan Tao, Liangliang Zhu, Lin Zeng, and Daohua Yao. 2012. "The Use of the Kurtosis Metric in the Evaluation of Occupational Hearing Loss in Workers in China: Implications for Hearing Risk Assessment." Noise and Health 14 (61): 330-42.

Deiters, Kristy K., Gregory A. Flamme, Stephen M. Tasko, William J. Murphy, Nathaniel T. Greene, Heath G. Jones, and William A. Ahroon. 2019. "Generalizability of Clinically Measured Acoustic Reflexes to Brief Sounds." Journal of the Acoustical Society of America 146 (5): 3993-4006.

Dunn, Derek E., Rickie R. Davis, Carol J. Merry, and John R. Franks. 1991. "Hearing Loss in the Chinchilla from Impact and Continuous Noise Exposure." The Journal of the Acoustical Society of America 90 (4): 1979-85. https://doi.org/10.1121/1.401677.

Erdreich, John. 1986. "A Distribution Based Definition of Impulse Noise.” The Journal of the Acoustical Society of America 79 (4): 990-98. https://doi.org/10.1121/1.393698.

Flamme, G. A., and William J. Murphy. 2020. The Noise Manual Chapter 6: Brief High Level Sounds. Edited by D. K. Meinke, E. H. Berger, R. L. Neitzel, D. Driscoll, and K. Bright. 6th ed. Fairfax, VA: AIHA Press.

Flamme, Gregory A., Kristy K. Deiters, Stephen M. Tasko, and William A. Ahroon. 2016. "Acoustic Reflexes Are Common but Not Pervasive: Evidence from the National Health and Nutrition Examination Survey, 1999-2012." International Journal of Audiology 56 (Supp 1): 52-62.

Goley, G. Steven, Won Joon Song, and Jay H. Kim. 2011. "Kurtosis Corrected Sound Pressure Level as a Noise Metric for Risk Assessment of Occupational Noises." The Journal of the Acoustical Society of America 129 (3): 1475-81. https://doi.org/10.1121/1.3533691.

Hamernik, Roger P., William A. Ahroon, Robert I. Davis, and Sheau-Fang Lei. 1994. "Hearing Threshold Shifts from Repeated 6-h Daily Exposure to Impact Noise." The Journal of the Acoustical Society of America 95 (1): 444-53. https://doi.org/10.1121/1.408338.

Hamernik, Roger P, James H Patterson, and Richard J Salvi. 1986. "The Effect of Impulse Intensity and the Number of Impulses on Hearing and Cochlear Pathology in the Chinchilla." J. Acoust.Soc.Am. 81 (4): 1118-29. 
Hanyu, Toshiki. 2014. “Analysis Method for Estimating Diffuseness of Sound Fields by Using Decay-Cancelled Impulse Repsonse.” Building Acoustics 21 (2): 125-33.

ISO. 2008. "ISO 3382:2:2008; Acoustics-- Measurement of the Reverberation Time-- Part 2: Ordinary Rooms." International Organization for Standardization. - 2019. "ISO 12913-3:2019; Acoustics-- Soundscape-- Part 3: Data Analysis." International Organization for Standardization.

Jeon, Jin Yong, Hyung Suk Jang, and Yong Hee Kim. 2013. "Subjective and Objective Evaluations of Scattered Sounds in Concert Halls." In Proceedings of Meetings on Acoustics. Toronto, Canada.

Jeong, Cheol-Ho, Jonas Brunskog, and Finn Jacobsen. 2013. "Room Acoustic Transition Time Based on Reflection Overlap." In Proceedings of Meetings on Acoustics, 19:1aAAa2 1-5. Montreal, Canada: Acoustical Society of America.

Jones, Heath G., Nathaniel T. Greene, and William A. Ahroon. 2019. "Human Middle-Ear Muscles Rarely Contract in Anticipation of Acoustic Impulses: Implications for Hearing Risk Assessments." Hearing Research 378 (July): 53-62.

Kamerer, Aryn M., Judy G. Kopun, Sara E. Fultz, Carissa Allen, Stephen T. Neely, and Daniel M. Rasetshwane. 2019. "Examining Physiological and Perceptual Consequences of Noise Exposure." The Journal of the Acoustical Society of America 146 (5): 3947-59. https://doi.org/10.1121/1.5132291.

Kardous, Chucri A., and Robert D. Willson. 2004. "Limitations of Using Dosimeters in Impulse Noise Environments." Journal of Occupational and Environmental Hygiene 1 (7): 45662. https://doi.org/10.1080/15459620490465839.

Kardous, Chucri A., Robert D. Willson, and William J. Murphy. 2005. "Noise Dosimeter for Monitoring Exposure to Impulse Noise.” Applied Acoustics 66 (8): 974-85. https://doi.org/10.1016/j.apacoust.2004.11.007.

Lei, Sheau-Fang, William A Ahroon, and Roger P Hamernik. 1994. "The Application of Frequency and Time Domain Kurtosis to the Assessment of Hazardous Noise Exposures." Journal of the Acoustical Society of America 96 (3): 1435-44.

Long, Marshall. 2014. Architectural Acoustics. 2nd ed. Waltham, MA: Elsevier Inc.

McGregor, Kara D., Gregory A. Flamme, Stephen M. Tasko, Kristy K. Deiters, and William A. Ahroon. 2017. "Acoustic Reflexes Are Common but Not Pervasive: Evidence Using a Diagnostic Middle Ear Analyser.” International Journal of Audiology 57 (Supp 1): S4250 .

Murphy, William J., and Chucri A. Kardous. 2012. "In-Depth Survey Report: A Case for Using a-Weighted Equivalent Energy as a Damage Risk Criterion." U.S. Department of Health and Human Services, Public Health Service, Centers for Disease Control and Prevention, National Institute for Occupational Safety and Health. https://doi.org/10.26616/NIOSHEPHB35011a.

Murphy, William J., and Ning Xiang. 2019. "Room Acoustic Modeling and Auralization at an Indoor Firing Range." The Journal of the Acoustical Society of America 146 (5): 386872. https://doi.org/10.1121/1.5132286.

Officials of the City of Omaha, Nebraska. 1996. Omaha Municipal Code Charter and General Ordinances of the City Volume I. Chapter 17.

Paiva Vianna, KarinaMary de, Rui ManuelCalejo Rodrigues, and MariaRegina Alves Cardoso. 2015. "Noise Pollution and Annoyance: An Urban Soundscapes Study." Noise and Health 17 (76): 125. https://doi.org/10.4103/1463-1741.155833. 
Patterson, James H. 1991. "Effects of Peak Pressure and Energy of Impulses." The Journal of the Acoustical Society of America 90 (1): 205-8. https://doi.org/10.1121/1.401289.

Price, G. Richard. 2007a. "Predicting Mechanical Damage to the Organ of Corti." Hearing Research 226 (1-2): 5-13. . 2007b. "Validation of the Auditory Hazard Assessment Algorithm for the Human with Impulse Noise Data." Journal of the Acoustical Society of America 122 (5): 2786-2802.

Qiu, Wei, Roger P Hamernik, and Bob Davis. 2006. "The Kurtosis Metric as an Adjunct to Energy in the Prediction of Trauma from Continuous, NonGaussian Noise Exposures." Journal of the Acoustical Society of America 120 (4): 3901-6.

Qiu, Wei, Roger P Hamernik, and Robert I. Davis. 2013. "The Value of a Kurtosis Metric in Estimating the Hazard to Hearing of Complex Industrial Noise Exposures." Journal of the Acoustical Society of America 133 (3): 2856-66.

Roberto, M., R. P. Hamernik, R. J. Salvi, D. Henderson, and R. Milone. 1985. "Impact Noise and the Equal Energy Hypothesis." The Journal of the Acoustical Society of America 77 (4): 1514-20. https://doi.org/10.1121/1.391993.

Smalt, Christopher J., Joe Lacirignola, Shakti K. Davis, Paul T. Calamia, and Paula P. Collins. 2017. "Noise Dosimetry for Tactical Environments." Hearing Research 349 (June): 42 54.

Sulkowski, W. J., S. Kowalska, and A. Lipowczan. 1983. "Hearing Loss in Weavers and DropForge Hammermen: Comparative Study on the Effects of Steady-State and Impulse Noise." In Proceedings ICBEN 1983, 171-84. Torino, Italy: ICBEN.

Suter, Alice H. 2017. “Occupational Hearing Loss from Non-Gaussian Noise." Seminars in Hearing 38 (3): 225-62.

Thiery, L., and C. Meyer-Bisch. 1988. "Hearing Loss Due to Partly Impulsive Industrial Noise Exposure at Levels between 87 and 90 DB(A)." The Journal of the Acoustical Society of America 84 (2): 651-59. https://doi.org/10.1121/1.396844.

U.S. Department of Defense. 2015. "Department of Defense Design Criteria Standard: Noise Limits." This standard establishes acoustic noise limits and prescribes testing requirements and measurement procedures for determining conformance to the noise limits stated herein. The standard specifies sound pressure level limits and measurement procedures to promote personnel safety, speech intelligibility, and security from acoustic detection and recognition. Acoustical noise limits for determining conformance to community annoyance requirements are beyond the scope of this standard. Noise limits for community annoyance are covered by local laws and statutes. DoD materiel (motor vehicles, construction and material handling equipment, mobile generator sets, and portable air compressors) may have to comply with these local laws and statutes anywhere in the world they operate.

U.S. Department of Labor. 2008. 1910.95 - Occupational Noise Exposure. | Occupational Safety and Health Administration. Occupational Safety and Health Standards. Vol. 1910.95. https://www.osha.gov/laws-regs/regulations/standardnumber/1910/1910.95.

U.S. National Institute for Occupational Safety and Health. 1998. "Criteria for a Recommended Standard: Occupational Noise Exposure.” U.S. Department of Health and Human Services.

Ward, W Dixon. 1968. "PROPOSED DAMAGE-RISK CRITERION FOR IMPULSE NOISE (GUNFIRE).” National Academy of Sciences National Research Council. 
Xie, Hong-wei, Wei Qiu, Nicholas J. Heyer, Mei-bian Zhang, Peng Zhang, Yi-ming Zhao, and Roger P. Hamernik. 2016. "The Use of the Kurtosis-Adjusted Cumulative Noise Exposure Metric in Evaluating the Hearing Loss Risk for Complex Noise." Ear and Hearing 37 (3): 312-23.

Zagadou, Brissi, Philemon Chan, Kevin Ho, and David Shelley. 2016. "Impulse Noise Injury Prediction Based on the Cochlear Energy." Hearing Research 342 (December): 23-28.

Zechmann, Edward Louis. 2019. "Characterization of Noise with Fractional-Octave-Band Filters and the Variance and Kurtosis of the Sound Pressure." Pennsylvania State University.

Zhao, YM, W Qiu, L Zeng, SS Chen, RI Davis, and RP Hamernik. 2010. “Application of the Kurtosis Statistic to the Evaluation of the Risk of Hearing Loss in Workers Exposed to High-Level Complex Noise.” Ear and Hearing 31 (4): 527-32. 


\section{Appendix A: MATLAB Code}

\section{A-1: Absorption Coefficient Calculation from Room Geometry and Reverberation Time (getAbsorptionCoef.m)}

This program contributed to all tables or graphs that include sound absorption coefficients; examples include Figure 4.2 and Table 4.1. As stated in the body of the thesis, it uses rearranged forms of the Sabine and Norris-Eyring equations for reverberation time to inform the user about which equation is more suitable for the room and what value it produces for the absorption coefficient.

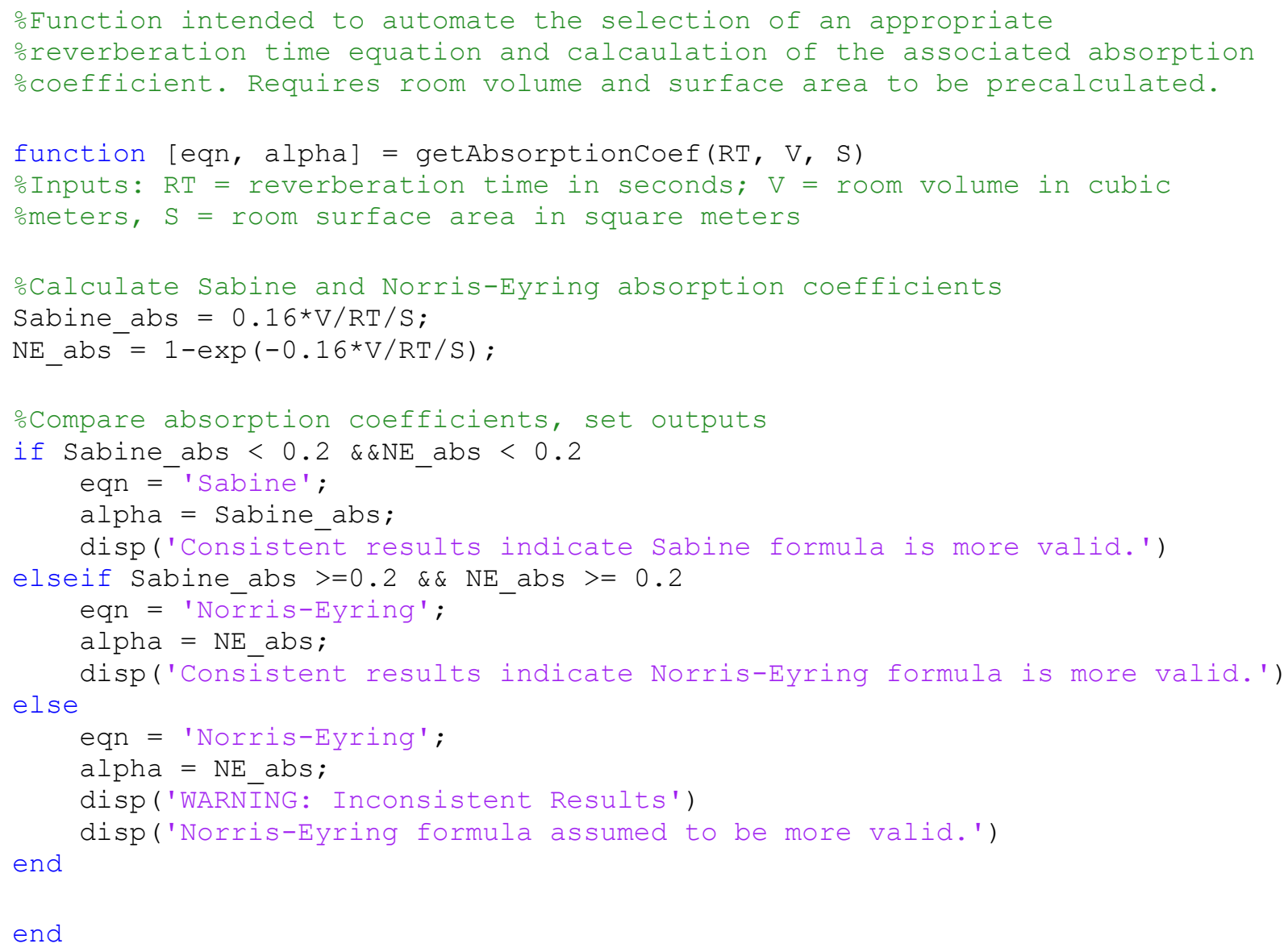




\section{A-2: Normalized Number of Peaks Calculation from Room Impulse Response (getNormedPeaks.m)}

This program contributed to all figures and tables which feature numbers of peaks;

examples include Figure 4.3 and Table 4.3. As stated in the body of the thesis, it takes impulse response data and counts the number of peaks within $20 \mathrm{~dB}$ of the highest peak present in the impulse response. The number of peaks associated with the anechoic "internal reference" measurement was indeed one, so the value of the variable "Norm_Const" (short for "normalization constant") is intentional.

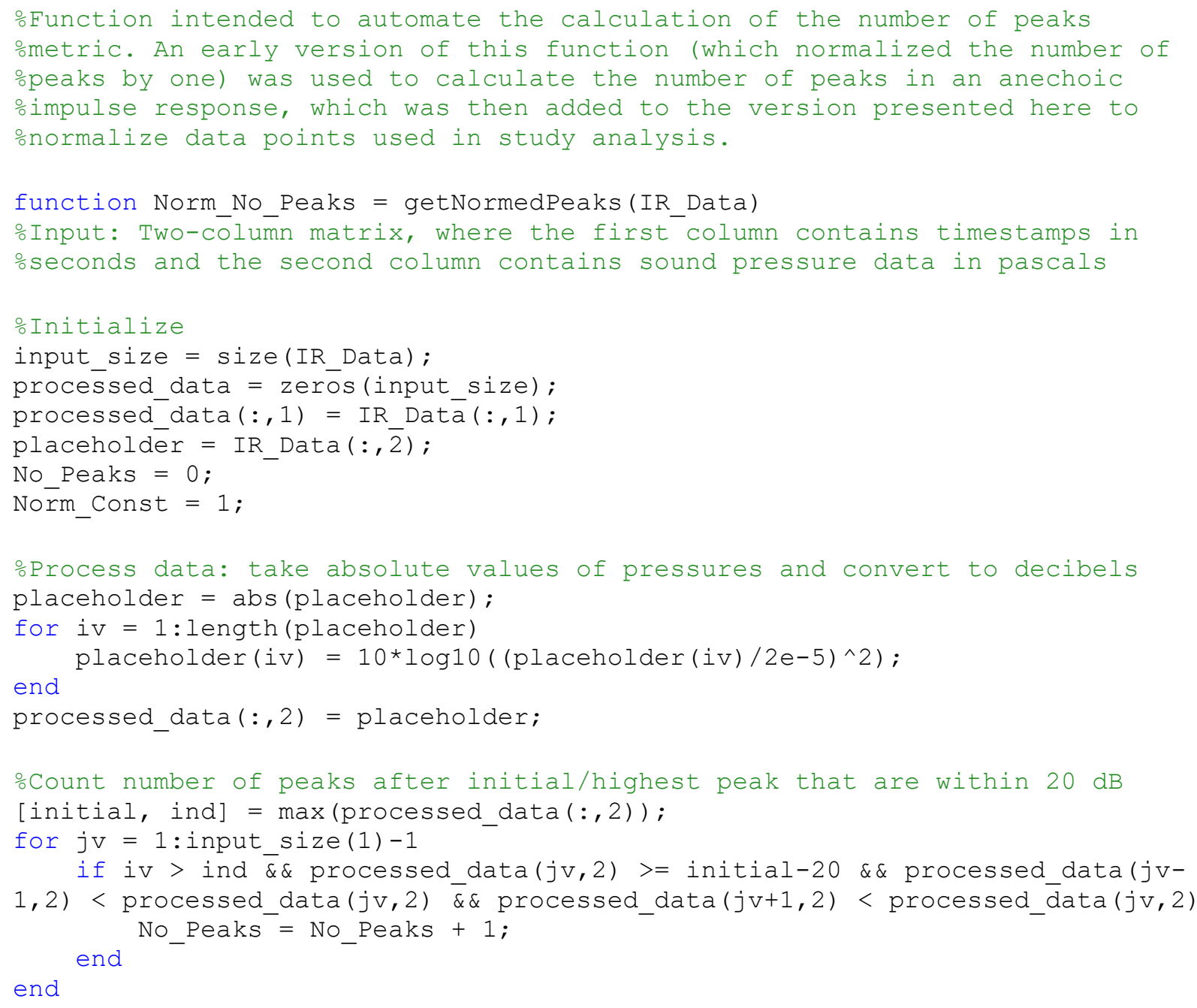


oCalculate normalized number of peaks Norm_No_Peaks = No_Peaks/Norm_Const;

end 


\section{A-3: Kurtosis Level Calculation from Convolution (getConvolvedLkFourSignals.m)}

This program contributed to all figures and tables that cite values for the kurtosis level.

Examples include Figure 4.2 and Table 4.4. As stated in the body of the thesis, it creates a sixtysecond signal at a $44.1 \mathrm{kHz}$ sampling rate for each sound of interest, then convolves each with the room impulse response input and returns a kurtosis level for the resulting signal.

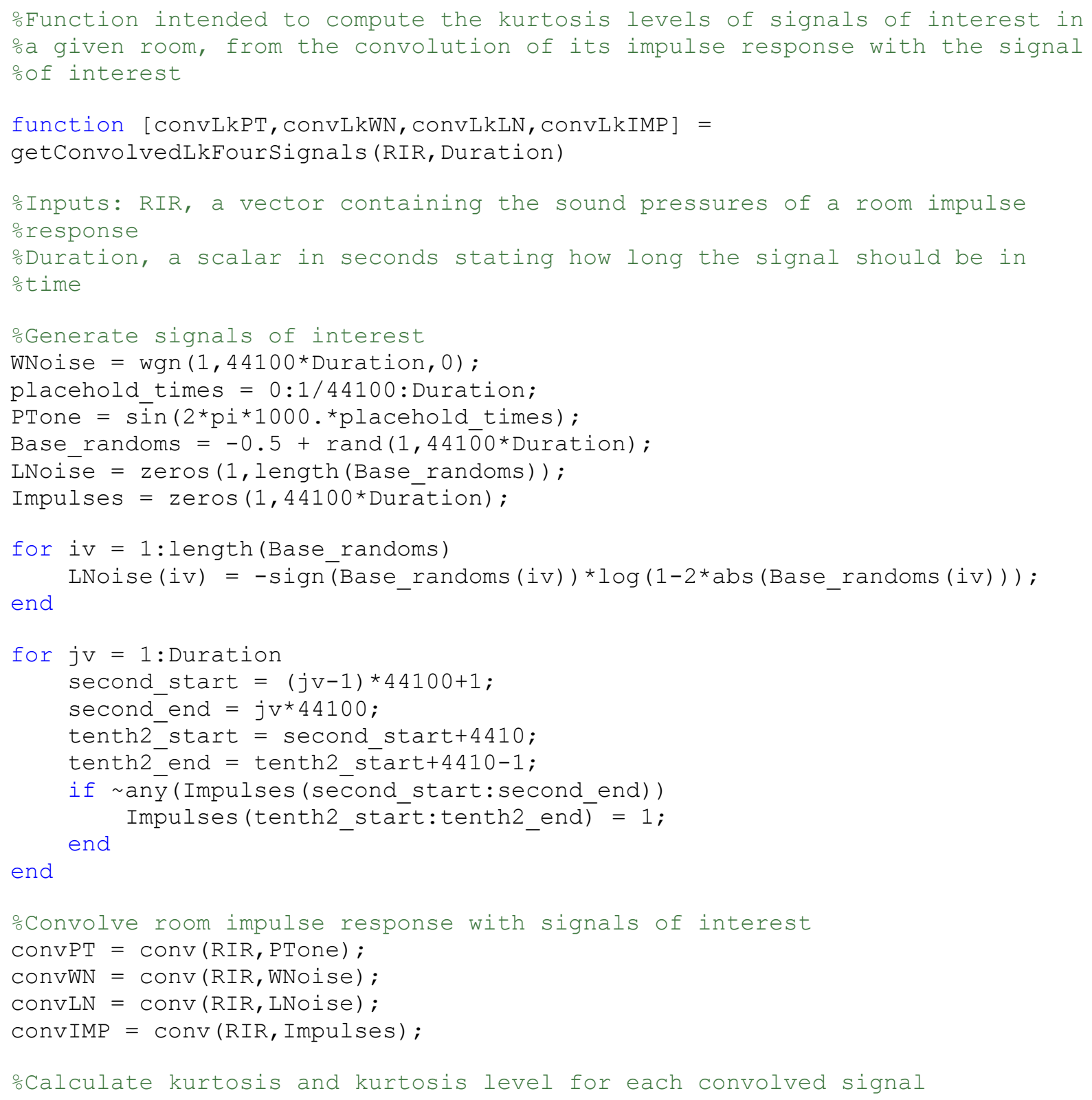




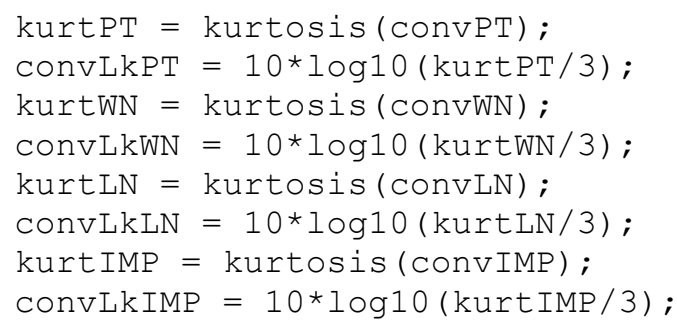




\section{Appendix B: Discussion of Original Methodology}

This study originally collected data for the calculation of kurtosis levels by using the Larson Davis Model 831 sound level meter and a signal generator connected to a loudspeaker to play the signals of interest defined by Zechmann into the rooms themselves; this methodology, despite using the best capabilities of the sound level meter and ensuring consistency among the signals played, created several problems for the analysis process. Firstly, while this method made it possible to approximate the exposure lengths used to calculate kurtosis levels of noise signals by other researchers, there was still information loss due to the use of the sound level meter's "time history" function rather than its sound recording capabilities. Although that choice was made to respect the privacy of people going about their business outside the rooms being tested, it also created discrepancies between the expected values stated by Zechmann and the internal benchmark values calculated using the study's own equipment in the Boys Town National Research Hospital anechoic chamber. Secondly, the speaker used for that portion of the data collection procedure was directional (unlike the omnidirectional loudspeaker used to collect impulse response data), which meant that it provided a different acoustical excitation to the room despite every effort being made to locate the two speakers close together and to point the directional speaker towards the room's center. This was an uncontrolled variable in a study that sought to understand how two specific acoustical properties of a given room would affect a procedurally sensitive metric.

Once these facts' negative impact on the data became apparent, procedures were changed to eliminate as much of said impact as possible. Fortunately, the changes opened more doors than they closed despite COVID-19 lockdown measures stopping data collection; they also 
resolved the aforementioned discrepancies to a large degree and simplified the study's methods for those seeking to understand or replicate them. 